\title{
A GENERIC DECISION MAKING FRAMEWORK FOR AUTONOMOUS SYSTEMS
}

\author{
A Thesis \\ Presented to \\ the Faculty of California Polytechnic State University \\ San Luis Obispo
}

\author{
In Partial Fulfillment \\ of the Requirements for the Degree \\ Master of Science in Computer Science
}

by

Connor Lange

June 2013 
(C) 2013

Connor Lange

ALL RIGHTS RESERVED 


\section{COMMITTEE MEMBERSHIP}

TITLE:

DATE SUBMITTED:

COMMITTEE CHAIR:

\author{
Professor \\ Computer Science Department
}

COMmittee Member: Dr. Phil Nico

Associate Professor

Computer Science Department

COMMITTEE MEMBER: Dr. Jordi Puig-Suari

Professor

Aerospace Engineering Department 


\begin{abstract}
A Generic Decision Making Framework for Autonomous Systems
\end{abstract}

\title{
Connor Lange
}

With the rising popularity of small satellites, such as CubeSats, many smaller institutions previously incapable of developing and deploying a spacecraft have starting to do so. Institutions with a history of space flight, such as NASA JPL, have begun to put projects on CubeSats that would normally fly on much larger satellites. As a result, the institutions with space flight heritage have begun to port spacecraft software that was previously designed for much larger and more complex satellites to the CubeSat platform.

Unfortunately for universities, who are the majority of all institutions developing CubeSats, these ported systems are too large and complex to be a practical control solution. Student teams have a high turnover rate due to graduation and when a student becomes an expert on the control system, they graduate; most students get a maximum of two or three years of experience before graduating.

This thesis proposes the Generic Decision Making Framework for Autonomous Systems (GDMFAS) as an accessible, easily extensible, component-based executive system architecture. The architecture is designed for Linux distributions, including the custom Linux distribution used by PolySat, and is implemented using $\mathrm{C}++$. The proposed framework provides much of the same functionality as systems designed for larger satellites in a smaller, more straightforward package, which includes both scheduling and executive components. This thesis also provides validation for the prototype implementation and evaluates the system according to six metrics. The metric analysis for this work is then compared with the metric analyses of previous works. 


\section{Acknowledgements}

There are many people that have helped make this thesis possible. Each one of them have improved my life and expanded my horizons in some way. Regardless of if I interacted with them a little or a lot, this work would not be possible without them.

My family and friends - Without their support and encouragement, this work would not have been possible.

Dr. Kurfess - For introducing me to the fields of artificial intelligence, expert systems, and usability, and for providing me with guidance and support throughout the development of this work.

Dr. Puig-Suari - For providing an opportunity for me to get experience with the design, implementation, and verification of a satellite system and for increasing my domain knowledge and providing valuable feedback.

PolySat and CubeSat - For working with me to develop and test satellites and groundstation software over the past few years. This thesis would not have been possible without the knowledge and interdisciplinary-experiences we have shared. 


\section{Contents}

List of Tables $\quad$ x

List of Figures $\quad$ xi

1 Introduction 1

1.1 Problem Framing . . . . . . . . . . . . . . . . . . . 1

1.2 GDMFAS Introduction . . . . . . . . . . . . . . . 4

1.3 Thesis Scope. . . . . . . . . . . . . . . 5

2 Literature Survey $\quad 7$

2.1 Comparison Metrics . . . . . . . . . . . . . . 7

2.1.1 Extensibility..................... 8

2.1.2 Domain Applicability . . . . . . . . . . . . . . . 8

2.1.3 System Overhead . . . . . . . . . . . . . 9 9

2.1.4 Mission Accuracy . . . . . . . . . . . . . . . 10

2.1.5 Error Recovery . . . . . . . . . . . . . . 10

2.1.6 Usability . . . . . . . . . . . . . . . . . 11

2.2 Review of Adaptable Systems . . . . . . . . . . . . . . . 11

2.2.1 The Completely Fair Scheduler . . . . . . . . . . . . . 12

2.2.2 The Lights-Out Ground Operations System . . . . . . . . 14

2.2.3 The Autonomous Satellite Operations Framework . . . . . 19

2.3 Review of Target Domain Systems . . . . . . . . . . . . . . 23

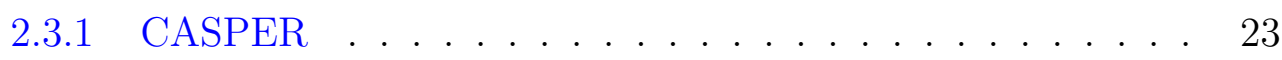

2.3.2 NEAT . . . . . . . . . . . . . . . . 28

2.3.3 Other Notable Systems . . . . . . . . . . . . . . . . 31 
2.4 Literature Summary . . . . . . . . . . . . . . . . . . . 31

3 GDMFAS Design 33

3.1 Definition of Tasks and Data Items _ . . . . . . . . . . . 34

3.2 Task Manager . . . . . . . . . . . . . . . . . . . . . . . . . 38

3.2 .1 Task Manager Overview . . . . . . . . . . . . . . . 38

3.2 .2 Adding a New Task . . . . . . . . . . . . . . . . 38

3.2 .3 Update of Task Properties . . . . . . . . . . . . . . . . 39

3.2.4 Relaying Task Status . . . . . . . . . . . . . . . . . . 40

3.3 Scheduler . . . . . . . . . . . . . . . . . . . . . . . . 41 4

3.3 .1 Risk Manager . . . . . . . . . . . . . . . . . . 43

3.3 .2 Prioritizer . . . . . . . . . . . . . . . . 44 4

3.3.3 Scheduling Algorithm Overview . . . . . . . . . . . . 46

3.4 System Monitor . . . . . . . . . . . . . . . . . . . . . . . 49

3.4 .1 System Safety . . . . . . . . . . . . . . . . . . 49

3.4.2 Resource Modelling . . . . . . . . . . . . . . . . . . . . . 49

3.4.3 Monitored Execution and the System Alert Level . . . . . 50

3.4.4 Scheduler Override . . . . . . . . . . . . . . . . . . . 52

3.4.5 Communication Module . . . . . . . . . . . . . . . 53

4 Implementation $\quad 54$

4.1 Framework Implementation . . . . . . . . . . . . . . . 54

4.1 .1 Overview . . . . . . . . . . . . . . . 54

4.1 .2 Resource Implementation . . . . . . . . . . . . . . . . 55

4.1 .3 Task Implementation . . . . . . . . . . . . . . . . . 56

4.1.4 Component Communication Implementation . . . . . . . 57

4.1.5 Task Manager Implementation _. . . . . . . . . . 58

4.1.6 Scheduler Implementation . . . . . . . . . . . . 58

4.1.7 System Monitor Implementation . . . . . . . . . . . . 59

4.2 Example Implementation . . . . . . . . . . . . . . . . . . 60

4.2.1 CPU Resource Implementation . . . . . . . . . . . . 60

4.2.2 Memory Resource Implementation . . . . . . . . . . . . . 61 
4.2.3 Current and Battery Resource Implementation . . . . . . . 61

4.2.4 Sample Task Sets . . . . . . . . . . . . . . . . 61

5 Validation $\quad 63$

5.1 Verifying Correctness . . . . . . . . . . . . . . . 63

5.2 Metric Evaluation . . . . . . . . . . . . . . . . 64

5.2.1 Extensibility.................. 65

5.2.2 Domain Applicability . . . . . . . . . . . . . . 66

5.2 .3 System Overhead . . . . . . . . . . . . . . . . 66

5.2 .4 Mission Accuracy . . . . . . . . . . . . . . 69

5.2.5 Error Recovery . . . . . . . . . . . . . . 69

5.2 .6 Usability . . . . . . . . . . . . . . . 70

6 Conclusion $\quad 74$

6.1 Conclusion . . . . . . . . . . . . . . . . . . 74

6.2 Future Work . . . . . . . . . . . . . . . . . 75

6.3 Thesis Contributions . . . . . . . . . . . . . 75

$\begin{array}{ll}\text { Bibliography } & 77\end{array}$ 


\section{List of Tables}

2.1 Metric Summary for the Completely Fair Scheduler . . . . . . . . 14

2.2 Metric Summary for LOGOS . . . . . . . . . . . . . . . . . . . . 19

2.3 Metric Summary for ASOF . . . . . . . . . . . . 22

2.4 Metric Summary of CASPER . . . . . . . . . . . . . . 28

2.5 Metric Summary of NEAT . . . . . . . . . . . . . . . 31

2.6 Literature Metric Summary . . . . . . . . . . . . . . . . 32

3.1 Properties of a Task . . . . . . . . . . . . . . . . 35

3.2 Properties of an Abstract Resource . . . . . . . . . . . . 36

5.1 Results for Unit Test Scenarios . . . . . . . . . . . . . . . . . 64

5.2 Metric Summary of GDMFAS . . . . . . . . . . . . . 73 


\section{List of Figures}

1.1 A 1 U CubeSat . . . . . . . . . . . . . . . . . 1

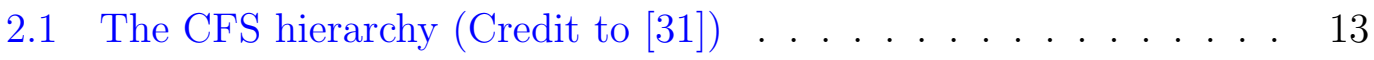

2.2 LOGOS Overview (Credit to [42]) . . . . . . . . . . . . 15

2.3 LOGOS Interactions (Credit to [42]) $\ldots \ldots \ldots \ldots \ldots$

2.4 ASOF Design Overview (Credit to [5]) . . . . . . . . . 20

2.5 The CASPER Architecture (Credit to [11]) . . . . . . . . . . 24

2.6 The EO-1 Architecture (Credit to [12]) $\ldots \ldots \ldots \ldots \ldots$

3.1 The scheduling process . . . . . . . . . . . . . . . . 42

3.2 A visual representation of the addTask function. Note that Tasks with absolute deadlines must begin by their deadlines. Although Task 3 had a higher priority than Task 1, it did not fit in its place. As a result, it is placed after the Task with an absolute deadline. . 47 


\section{Chapter 1}

\section{Introduction}

\section{$1.1 \quad$ Problem Framing}

With the rise of small satellites, such as CubeSats $[19]^{1}$ (depicted in Figure 1.1), many institutions are attempting to complete missions on small satellites that were previously reserved for much larger spacecraft [35]. Many institutions

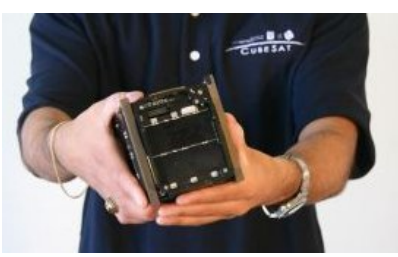

Figure 1.1: A 1U CubeSat

making this change, such as NASA JPL, have very complex control systems ${ }^{2}$ that have been validated on previous missions and are uniquely suited for their satellite's operational needs. However, the majority of satellite development efforts have not been completed by the government or private sector; they have

${ }^{1} 1000 \mathrm{~cm}^{3}$ for a $1 \mathrm{U}$ satel $\mathrm{li}$ te

${ }^{2}$ CAS PER i s presen ted i n Chapter 2 
been completed by university satellite development teams [24], such as Cal Poly's PolySat [34].

PolySat, like many other university satellite teams, is comprised of a multidisciplinary group of students. None of the student developers are experts in their domain and many do not have the skills to develop certain aspects of the satellite (such as wireless communication software) until they have taken the appropriate classes or have taken it upon themselves to learn the material. By the time these students gain domain knowledge and learn all the skills required to contribute fully, they graduate; the average PolySat developer is only around for 2 to 3 years.

As a result of the high turnover, many teams have used or adapted existing systems in an attempt to decrease development time. A recent successful example of this is PolySat's move from a PIC micro-controller to an ARM processor running a custom Linux image. This transition allowed PolySat software developers to directly apply knowledge learned in systems programming and operating systems courses to satellite development, decreasing the learning curve [28]. While the adoption of a Linux-based architecture added multiple layers of faulttolerance [28], it didn't provide the same oversight an executive system provides. While Linux has an out of memory (OOM) killer and allocates CPU usage via the scheduler, there is no insight into other satellite resources (solar panels, cameras) by default.

Executive systems offer a unique opportunity for university-developed CubeSats that is not likely to be needed in larger, more experienced development efforts from other institutions such as NASA or Northrop Grumman. Since universitydeveloped CubeSats utilize a small group of inexperienced students (relative to their industry counterparts) and often have an accelerated timeline, software sys- 
tems inevitably isn't as robust as they could be. Additionally, although the overall system functions correctly, errors due to radiation effects (latching, bit-flips) and bugs are not handled. This can lead to an unknown state in the satellite and cause problems. Larger institutions typically spend a lot of money on RadHard components and extensive testing. For universities, $\$ 21,000$ [1] out of their total $\$ 200,000$ [38] budget for a single processor is a lot of money. Since many CubeSats are used for learning purposes and don't need to be near-invincible, most CubeSats use Commercial, Off The Shelf (COTS) parts, which are not RadHard. Further, it is usually not feasible to put CubeSats through bullet-proof software testing due to time constraints; students are typically juggling multiple assignments, studying, and participating in other extra-curricular activities. Unlike institutions in industry, PolySat software developers are volunteering to develop a satellite (sometimes multiple satellites) in their spare time. As a result of not dedicating 40 hours a week to the project and being on an accelerated development schedule, there simply isn't time for bullet-proof software testing and some bugs make it through the cracks.

While many other systems that provide similar functionality exist ${ }^{3}$ to the system proposed in this work, many of them have been designed for larger, more complex spacecraft with a significantly lower fault-tolerance [11] [32] [39]. While these systems can generate extremely detailed schedules and handle complicated errors, they do so at the cost of being large and unwieldy for those not intimately familiar with the system. Though the advanced scheduling and error resolution may be desirable for expensive, long-lasting spacecraft (the Glory satellite was supposed to operate for three years and cost $\$ 388$ million [29]), the level of faulttolerance, and the resulting complexity, can be overkill for a CubeSat, which

\footnotetext{
${ }^{3}$ di scussed i n Chapter 2
} 
typically cost between $\$ 50,000$ and $\$ 200,000$ [38], operate for between 3 and 9 months [23], and weigh no more than $1.33 \mathrm{~kg}$ per $1 \mathrm{U}$ [14]. In addition to the lower fault-tolerance requirements, Cal Polyś missions typically have action deadlines with a granularity of a millisecond or more; with mission deadlines such as activating an experiment at $\mathrm{T}+60$ days, or starting to record data at $\mathrm{T}+15$ seconds, the extremely precise, real-time scheduling and planning systems are not needed. Furthermore, most of those systems are proprietary, ITAR controlled [43], or both. This makes them harder to get access to and deploy.

Although existing spacecraft executive systems may be overkill, the general features they provide are not. However, for these features to be actively utilized by students developing CubeSats, they must be easy to understand, employ technology students will be familiar with, and have a minimal learning curve. Additionally, it is desirable for such systems to be self-reliant. Previous systems such as ASOF [5], made the assumption that the then-current GENSO [17] system would increase performance, although that system is now inactive.

\subsection{GDMFAS Introduction}

To solve the above problems, and make the system more fault tolerant, this thesis proposes the Generic Decision Making Framework for Autonomous Systems (GDMFAS), an accessible executive system architecture. GDMFAS significantly reduces the implementation overhead of a completed executive system by offering a full-featured framework users can build off of. GDMFAS supplies an API for autonomous system developers, which handles most core "brain" functions of the system without making the developers reinvent the wheel and implement it themselves. Basic versions of many time consuming tasks such as implementing 
predictive resource modelling, scheduling tasks, and getting overall system health and progress are already provided. The GDMFAS system provides three components: one to manage all the Tasks in the system (the Task Manager), one to schedule them and ensure all mission dead lines are met (the Scheduler), and one to monitor the Tasks as they execute in order to correct any misbehaviour and track the health of the satellite (the System Monitor). All the functionality provided by GDMFAS is done in a generic way, allowing GDMFAS to be applied to many different systems ranging from CubeSats, the target deployment, to a groundstation-spacecraft hybrid deployment.

\subsection{Thesis Scope}

Because of the near infinitely expandable nature of an abstract decision making framework, it is important to define the scope of such a framework. The scope of the framework discussed in this thesis is fairly expansive, but clearly defined. This work provides high-level explanations of all modules included in the framework, defines the data items required for such a framework, and discusses the prototype framework implementation. Additionally, implementation details for an example system built using the GDMFAS prototype is provided.

The description of the example system makes no attempt to assert the provided implementation details (such as scheduling algorithm or resource tracking methods) are the optimal ones; the example implementation is for validation and demonstration purposes. As a result of being a prototype, the implementation of the framework does not completely match the design outlined in this work; the design includes functionality that should be implemented or extended by the users of the framework, while the prototype offers a proof-of-concept. Despite the 
lack of implementation for some of the features, this work does present an analysis of the potential benefits and drawbacks of some of the outlined approaches. 


\section{Chapter 2}

\section{Literature Survey}

\subsection{Comparison Metrics}

Before discussing the existing work in the literature, it is important to first outline the metrics that will be used to evaluate these works. These metrics summarize the design principles of the Generic Decision Making Framework for Autonomous Systems, and will evaluate how existing systems compare to the GDMFAS. The metrics are listed below:

1. Extensibility

2. Domain Applicability

3. System Overhead

4. Mission Accuracy

5. Error Recovery

6. Usability 
Each of the metrics will be scored with one of the following values:

- Very Poor

- Poor

- Moderate

- Good

- Very Good

The actual scoring of metrics attempts to be objective, but due to the nature of related systems, the implementation details and performance information are sometimes unavailable and the evaluation must rely on a thoughtful, although subjective, evaluation of the documents that are provided.

\subsubsection{Extensibility}

In the context of this work, Extensibility measures the degree to which a system can be built upon or expanded. If the system is generic and includes a high degree of abstraction, it would receive a high score under this metric. On the other hand, if the system was very narrowly designed with one project in mind, and all the components of the system were tightly coupled, the system would receive a low score.

\subsubsection{Domain Applicability}

Domain applicability refers to the degree to which a system is intended or designed for the target domain of this work (small, embedded autonomous systems). If the system was designed with a different intent, but could be easily 
adapted into a viable system in the target domain, it could still receive a decent score. For example, an operating system scheduler can still schedule processes in the target domain, but any other information about autonomous system specific resources, such as fuel, would not be considered. This metric primarily exists as a measure of how useful each system would be on a CubeSat.

\subsubsection{System Overhead}

In the context of this work, System Overhead refers to the effect of the reviewed system on the operation of the autonomous system. If the system uses significant resources, that system would receive a low score in this area. For example, a system that does a lot of complex calculations or stores a substantial amount of data on disk or in memory would get a low score for this metric. After

all, it is the job of a system similar to GDMFAS to improve the function of the overall autonomous system, not hinder it.

In an attempt to objectively assess the overhead of a system, the mappings below between average CPU use and a metric score will be used:

- $0 \% \leq C P U \leq 10 \%$ - Very Good

- $10 \%<C P U \leq 25 \%-$ Good

- $25 \%<C P U \leq 35 \%$ - Moderate

- $35 \%<C P U \leq 50 \%$ - Poor

- $50 \%<C P U$ - Very Poor

When these numbers are unavailable, a best-effort estimation of these values will be attempted based on design details, algorithms, and other information 
present in the literature.

\subsubsection{Mission Accuracy}

Mission accuracy refers to how well the reviewed system can meet mission deadlines. A system which poorly schedules tasks in a way where absolute deadlines can be missed would receive a poor score for this metric. To award scores for this metric, the following scale will be used:

- Very Good - All absolute deadlines are met, tasks are scheduled optimally, and real-time deadlines can be met.

- Good - All absolute deadlines are met and tasks are scheduled optimally

- Moderate - All absolute deadlines are met

- Poor - Absolute deadlines can be missed, but most mission objectives are completed on time

- Very Poor - Absolute deadlines can be missed and the system is not guaranteed to complete the mission.

\subsubsection{Error Recovery}

This metric refers to how well the reviewed system handles errors that arise in scheduled tasks during system operation; also included in this metric is how well the system monitors resource levels and what how intelligent the actions it takes are when these levels are insufficient. For example, a system that does not monitor resources and does no error handling (scheduled tasks just crash and aren't restarted) would receive a low score for this metric; a system that 
accurately monitors resources and intelligently restarts or reschedules a task in an error situation would receive a high score for this metric.

\subsubsection{Usability}

This metric refers to how easy it is for a new developer who is not a domain expert to get the reviewed system up and running. While this metric is somewhat subjective, in the context of this work, Usability refers to a small set of evaluable criteria. The first criterion is the degree to which the system must be configured before use. Significant configuration decreases the score awarded for this metric. This configuration may manifest itself in the form of configuration files, rules, or command languages. The second criterion is how difficult it is for an the system to be ported to the target domain. With ever changing small satellite technology, it is important for the system to be easily movable between architectures. A simple recompile would result in a high score, while changing libraries and modifying code would result in a very low score. The third and final criterion is how difficult the system is to understand for new users; proprietary intermediate systems and poor documentation (making configuring and extending the system difficult) contribute to a poor score for this metric.

\subsection{Review of Adaptable Systems}

This section provides a review of related systems that were not designed with the target domain specifically in mind but could be adapted or used as is in the target domain. Many of the related systems in this category only implement a subset of the components in GDMFAS. Systems that require modifications to source 
code or significant modification of the target system are not included.

\subsubsection{The Completely Fair Scheduler}

\section{Overview}

A common source of process/task scheduling solutions in the literature is operating system research and development. The Completely Fair Scheduler (CFS), the 2.6.23 Linux kernel scheduler, is a modular scheduling system that attempts to provide each task with a fair share of the operating system's resources; in this case, the resource is CPU time. In order to store tasks, CFS uses redblack trees to ensure $\mathrm{O}(\log \mathrm{N})$ operation. While the previous 2.6 Kernel scheduler operated in $\mathrm{O}(1)$ time, the heuristics the scheduler used to analyze processor usage were more complex than they needed to be. While the CFS may operate slower in the worst-case, it is much more extensible and less complex than the $\mathrm{O}(1)$ scheduler [22].

\section{Metric Analysis}

The primary benefit of the CFS is that it can be used as is for no extra cost on any system running the 2.6.23 Linux kernel. Due to the built-in nature of the CFS, the overhead is effectively zero since any system running the default 2.6.23 kernel will also be running the CFS; there is no additional burden above and beyond normal kernel operation. For this reason the CFS receives a Very Good score for System Overhead. Because the CFS is built into the kernel, it has no inherent knowledge of the target domain. While the scheduler will manage tasks that have already been started, it has no knowledge of the mission timeline and cannot accurately ensure that scheduled tasks are completed at the correct time 
on the mission timeline. These properties give the CFS a Very Poor score for Mission Accuracy. Additionally, the CFS is unaware of mission-specific resources that [39] [39] [11]tasks may be monitoring (such as the resources of a payload). The limited awareness of the CFS, combined with its non-existent recovery procedure upon a task failure, result in poor error recovery abilities. While the error recovery abilities may be poor, crashed or malfunctioning processes do not bring down the whole system, giving the CFS a Poor score for Error Recovery. With the increased prevalence of the Linux operating system on embedded boards in the target domain [28], the potential for using the CFS has increased; given CFS's issues with the target domain outlined above, the CFS only receives a Moderate score for the Domain Applicability Metric. Since the Linux kernel is open source [30] and readily available, developers that wish to add a new module to the scheduler can do so. The modular design and hierarchy of the scheduler system (shown in Figure 2.1 below) also contribute to the CFS's score of Good for Extensibility.

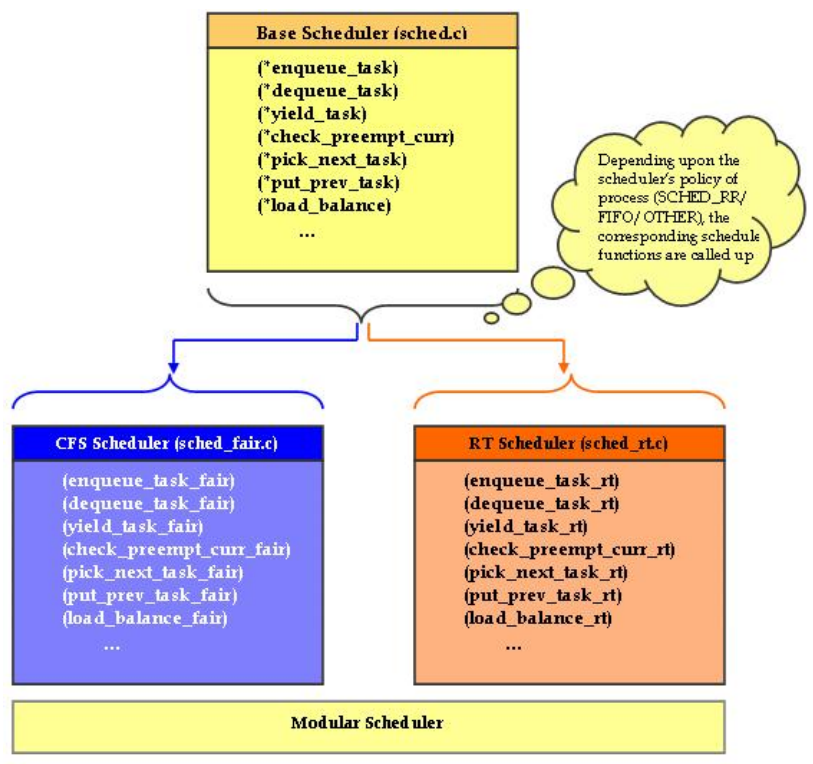

Figure 2.1: The CFS hierarchy (Credit to [31]) 
Since the Linux operating system's inner-workings (typically including the scheduler) is a very common topic in Computer Science and Computer Engineering curriculum [7] [36], many of the users of the CFS will be familiar with its general design and implementation. Since the CFS is part of the Linux kernel, the core scheduler requires no configuration. Finally, since the CFS has no proprietary modules and decent documentation, it receives a score of Very Good for the Usability metric.

Table 2.1: Metric Summary for the Completely Fair Scheduler

\begin{tabular}{|c|c|}
\hline Metric & Score \\
\hline Extensibility & Good \\
\hline Domain Applicability & Moderate \\
\hline System Overhead & Very Good \\
\hline Mission Accuracy & Very Poor \\
\hline Error Recovery & Poor \\
\hline Usability & Very Good \\
\hline
\end{tabular}

\subsubsection{The Lights-Out Ground Operations System}

\section{Overview}

The Lights-Out Ground Operations System (LOGOS) is a groundstation system developed in Java that utilizes a community of agents in an attempt to automate spacecraft operation; the system was originally developed by NASA's Goddard Space Flight Center in 1995. To automate the spacecraft during nominal conditions, LOGOS utilizes a previously developed expert system, the Generic Inferential Executor (GENIE); like the rest of the components in the system, GE- 
NIE operates as an agent in the agent community [42]. These agents collaborate between themselves and external modules to gather spacecraft data and make control decisions. An overview of this interaction is provided in Figure 2.2.

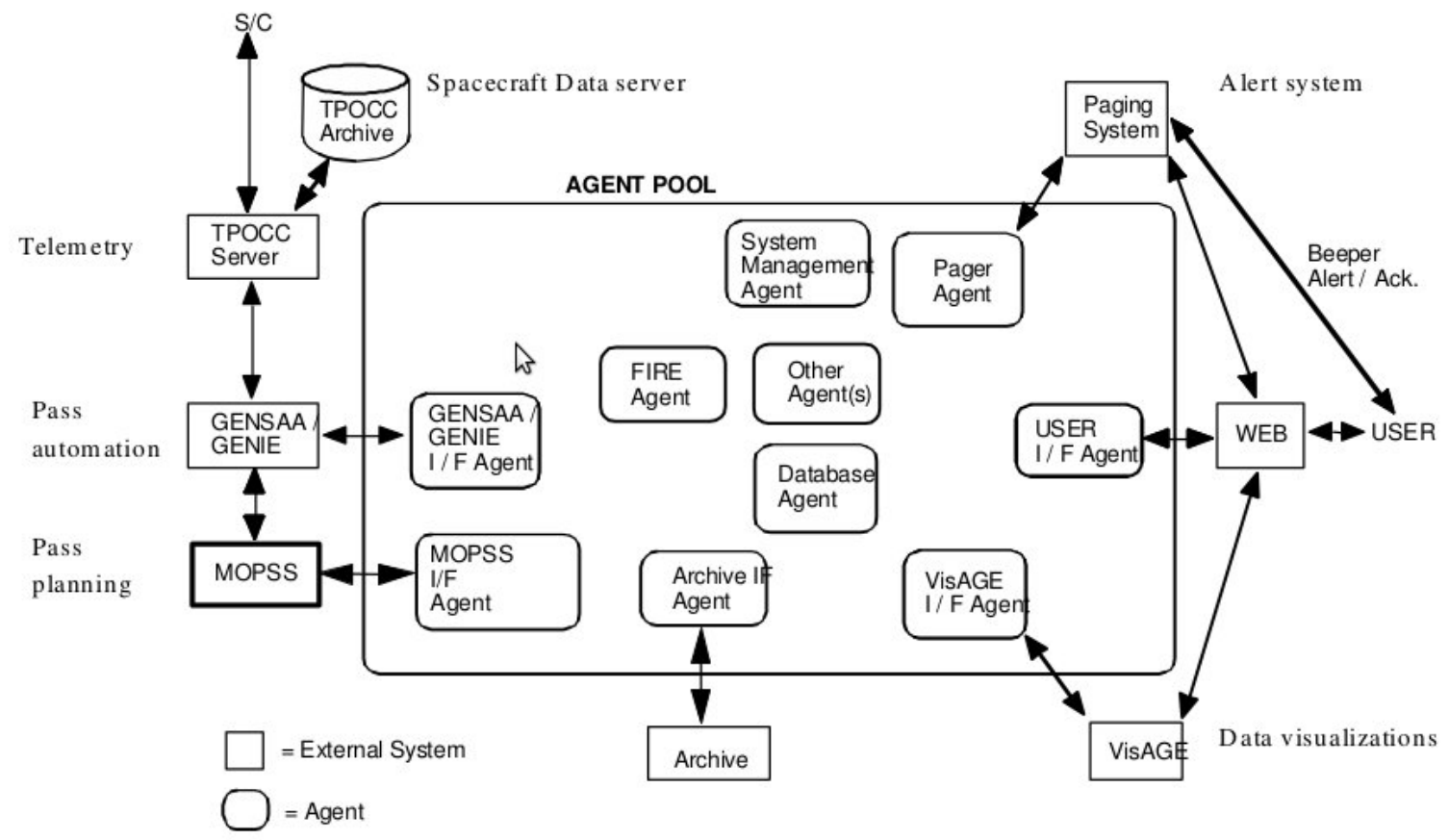

Figure 2.2: LOGOS Overview (Credit to [42])

In the off-nominal case where an error in space craft operation or commanding occurs, the agents in the community work together in an attempt to address the anomaly. In the event that the agent community cannot resolve the issue, LOGOS establishes a connection to a human analyst for assistance. The human operator can then work amongst the agents using the Agent Communication Language (ACL) to resolve the issue. In the most recent version of LOGOS, the ACL is the 
Knowledge Query and Manipulation Language (KQML) [42]. This interaction is shown below in Figure 2.3.

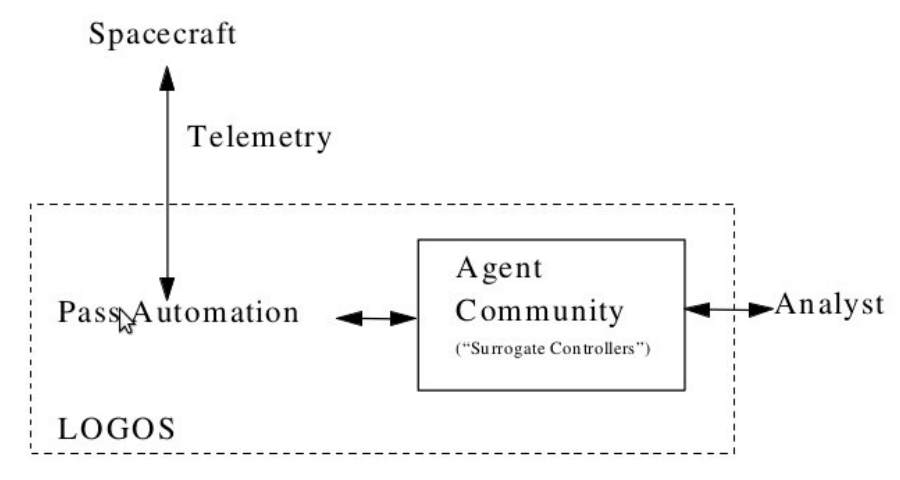

Figure 2.3: LOGOS Interactions (Credit to [42])

\section{Metric Analysis}

Like the CFS, LOGOS is a modular system and is easily extensible. However, LOGOS provides many more modules than just a scheduler and can include other systems as unique agents in the agent community. This level of abstraction and the generic nature of the LOGOS provides a simple interface for developers to create new modules. Any new modules can automatically interact with existing agents as long as the ACL is implemented by the new agent. For this reason, the LOGOS receives a score of Very Good for the Extensibility metric. Since the LOGOS was intended for deployment on spacecraft groundstations, it has a higher degree of Domain Applicability than the CFS. However, since the system was not intended for deployment on a spacecraft, it only receives a Good score for the Domain Applicability metric. 
While no detailed performance analysis of LOGOS exists in the literature, the System Overhead metric can be determined for a ported version of LOGOS based on a number of properties of the system. First, the LOGOS is developed in Java, an interpreted language [42]. While the project could be compiled to native machine code for use on embedded targets using GCJ [40], this method requires an additional 16MB of space for the glibc library needed by GCJ [37]. To conserve space, a small JVM (with sizes ranging between 200kB [27] and less than $1 \mathrm{kB}[10])$ could be deployed, although this approach would incur the performance hits associated with the JVM [16]. In addition to the Java issue, the communication between agents in the agent community will also incur a performance penalty. Depending on the number of agents and frequency of communication, the performance hit could be significant. As implemented, there are not enough agents (currently less than 20) to cause a significant penalty, although this can change as the system expands. With the original system, the round trip time (RTT) for communication between the groundstation and the spacecraft could cause performance issues if the delay was significant; however, since this analysis focuses on a ported version of the LOGOS, RTT is no longer a concern because the LOGOS would be operating on the spacecraft. Because there are a few performance issues with the LOGOS, but these issues do not overwhelm the system, the LOGOS receives a Moderate score for the System Overhead metric.

Since the LOGOS uses GENIE to analyze telemetry and conduct passes [42], the score for the Mission Accuracy metric is largely based on GENIE. GENIE has a mission timeline and tasks with absolute deadlines can be scheduled; whether the tasks with absolute deadlines have their deadlines met is up to the rules specified by the human operator [41]. Because the system can meet mission deadlines but cannot guarantee they are executed in a timely manner, the GENIE 
system meets the critera for a Good score for the Mission Accuracy metric; if the system misses a deadline it is due to the rules GENIE is using rather than an inherent issue with the system. Although GENIE has no error recovery abilities [41], LOGOS includes FIREAgent, an agent that diagnoses and resolves faults in the system [42]. However, this agent has limited knowledge, does not learn from its mistakes, and may request outside assistance from a human, incurring communications costs and significantly slowing down the schedule. Due to the above reasons, the LOGOS is awarded a score of Poor for the Error Recovery metric.

In terms of the Usability metric, the LOGOS has greatly varying subscores within the metric. For example, the LOGOS does not appear easy to port to the target domain; the code must either be ported to $\mathrm{C}++$ or changed so it is suitable as an embedded Java deployment. The port must also include the elimination of the intermediate communication link layer as it would no longer be a groundstation system. In addition to the efforts required to port the system, there is significant configuration required to use the LOGOS; GENIE must be configured with a set of rules, and each agent must have its behavior, agents it will communicate with, and its role in the system defined. The only redeeming quality of the LOGOS in terms of the Usability metric is the documentation for the project. Shortly after the project's development in 2000, there were a plethora of project documents providing requirements, design decisions, and Javadocs for the system [6]. Unfortunately, these documents have since disappeared and cannot be of any use to new users of the system. For the above reasons, the LOGOS receives a score of Very Poor for the Usability metric. 
Table 2.2: Metric Summary for LOGOS

\begin{tabular}{|c|c|}
\hline Metric & Score \\
\hline Extensibility & Very Good \\
\hline Domain Applicability & Good \\
\hline System Overhead & Moderate \\
\hline Mission Accuracy & Good \\
\hline Error Recovery & Poor \\
\hline Usability & Very Poor \\
\hline
\end{tabular}

\subsubsection{The Autonomous Satellite Operations Framework}

\section{Overview}

The Autonomous Satellite Operations Framework was developed in 2010 by Jason Anderson. Like LOGOS, the Autonomous Satellite Operations Framework (ASOF) was designed to be deployed on a spacraft groundstation and is a modular system. The ASOF is composed of five different components: the Agent, the Knowledge Base (KB), the Terminal Node Controller (TNC), the Line of Sight Executive (LOSE), and the Task File. The Agent is responsible for the transmission and receipt of uplink and downlink packets. The Knowledge Base is the framework component that creates an autonomous system for a particular satellite, while the Task File defines the tasks to be completed. To complete these tasks, the TNC module takes control of the groundstation TNC once the LOSE module determines that the satellite is available for communication [5]. A summary of the design is shown in Figure 2.4 below. 


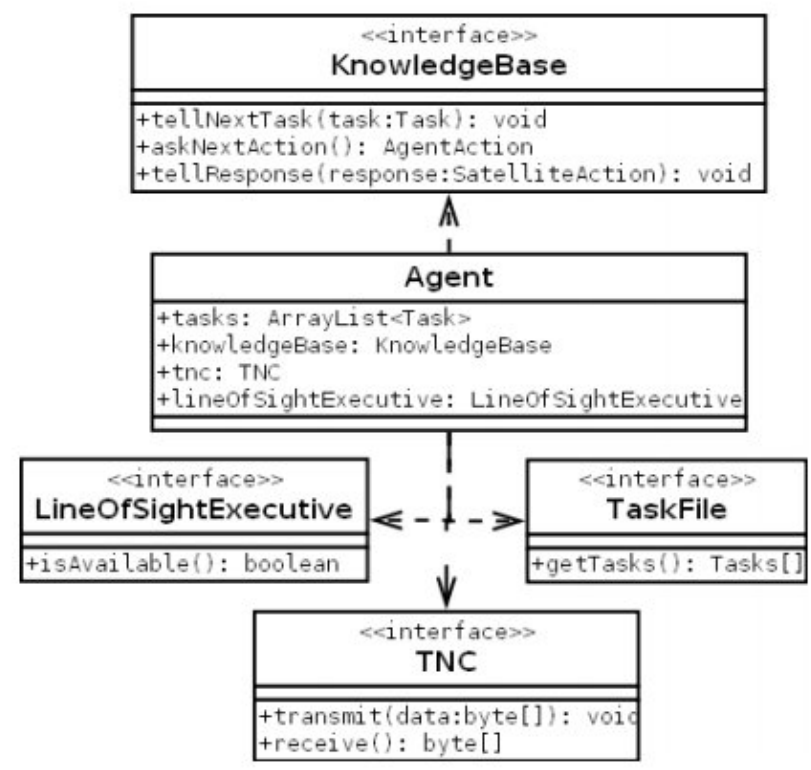

Figure 2.4: ASOF Design Overview (Credit to [5])

\section{Metric Analysis}

Although ASOF doesn't have the ability to add functionality in the form of additional agents in an agent pool, ASOF is no less Extensible than LOGOS. Like LOGOS, ASOF is also written in Java and has a well-defined API. Because of the object oriented nature of Java, multiple different agents can be derived from a single base class and generic agents can be expanded into more specific ones. Additionally, ASOF implements support for multiple different external interfaces (such as the GENSO groundstation network). Due to the generic nature of the framework and the multiple levels of abstraction, ASOF receives a score of Very Good for the Extensibility metric.

Like LOGOS, ASOF is primarily a groundstation system; although ASOF also has a separate module encapsulating the communication link, the smaller number of modules and the larger amount of functionality concentrated in each module 
results in a tighter coupling between the external groundstation functionality and ASOF. As a result, ASOF receives a slightly lower score than LOGOS, Moderate, for the Domain Applicability Metric.

Because ASOF is also written in Java, it shares the same performance issues as LOGOS (discussed in Section 2.2.2). Since ASOF has less agents communicating with each other than LOGOS, the System Overhead of ASOF due to inter-agent communication is lower than LOGOS. Further, the provided implementations utilize an expert system, a DFA model, or both in a Hybrid setup. Based on the implementation analysis, algorithm descriptions, and validation information from [5], ASOF has a lower execution overhead than LOGOS. As a result of this analysis, the ASOF system receives a score of Good for the System Overhead metric.

In all three implementations of ASOF agents, the mission timeline (or a set of "agendas") is formally defined in some form (DFA, RBS, or Hybrid). Since the agendas are set, the only reason the agenda would not execute in time would be due to an error on the satellite. In the RBS and Hybrid implementations, the error would be resolved and the agenda would continue executing. Although there is a slight delay to resolve the error, this would be the case with any intelligent system that couldn't prevent the error from occurring. Therefore, ASOF receives a Very Good score for the Mission Accuracy metric.

When an error occurs in ASOF, it may or may not be handled, depending on the implementation used. The DFA, RBS, and Hybrid implementations are capable of handling errors they are aware of, while only the RBS and Hybrid approaches have generic error handling abilities. Assuming the most capable ASOF implementation is used with a complete Knowledge Base, ASOF receives a Good for the Error Recovery metric. 
While ASOF is a very capable system in terms of Mission Accuracy and Error Recovery, it falls short in Usability. The RBS implementation requires significant preparation time to build out the Knowledge Base (on the order of weeks); this construction is a tedious process that can only be completed by existing satellite operators; even when the process is completed, the rule set may be incomplete [5]. If the user wanted to omit the rule-based error recovery portion of ASOF, the DFA implementation could be used; this implementation would score higher since it requires very minimal configuration, although it is less relevant to this work. Even with the use of the Hybrid implementation, the Knowledge Base construction is still required. The requirement of a Knowledge Base means that JESS is tightly coupled with the system, a characteristic that makes ASOF more difficult to port to an embedded environment. As with LOGOS, ASOF is heavily documented. The design, implementation, and validation efforts are clearly presented and components of the system are easily accessible. ASOF's clear documentation and easily accessible components bring ASOF's score for the Usability metric above LOGOS', up to a Moderate score.

Table 2.3: Metric Summary for ASOF

\begin{tabular}{|c|c|}
\hline Metric & Score \\
\hline Extensibility & Very Good \\
\hline Domain Applicability & Moderate \\
\hline System Overhead & Good \\
\hline Mission Accuracy & Very Good \\
\hline Error Recovery & Good \\
\hline Usability & Moderate \\
\hline
\end{tabular}




\subsection{Review of Target Domain Systems}

This section provides a review of related systems that were designed for the target domain, small spacecraft. Like the previous set of systems, some of the reviewed systems may not implement all the features of GDMFAS.

\subsubsection{CASPER}

\section{Overview}

The Continuous Activity Scheduling, Planning, Execution, and Replanning system (CASPER) [11] is a scheduler developed by JPL in 1999. CASPER has been deployed as part of many different systems, including Earth Observer 1 (EO1) [12] and IPEX [25], one of Cal Poly's upcoming CubeSats. In these systems, CASPER operates as a scheduler and planner that generates a schedule based on mission constraints and spacecraft resources. In the event of an error, CASPER is also capable of replanning the schedule to resolve the issue. The CASPER architecture is shown below in Figure 2.5.

On EO-1, CASPER constructed a detailed schedule for the next day's operations, and generated an abstract schedule for the rest of the week [12]. Although CASPER provides a range of functionality, it is typically deployed in conjunction with other systems. An example architecture that includes CASPER (EO-1's architecture) is shown below in Figure 2.6. 


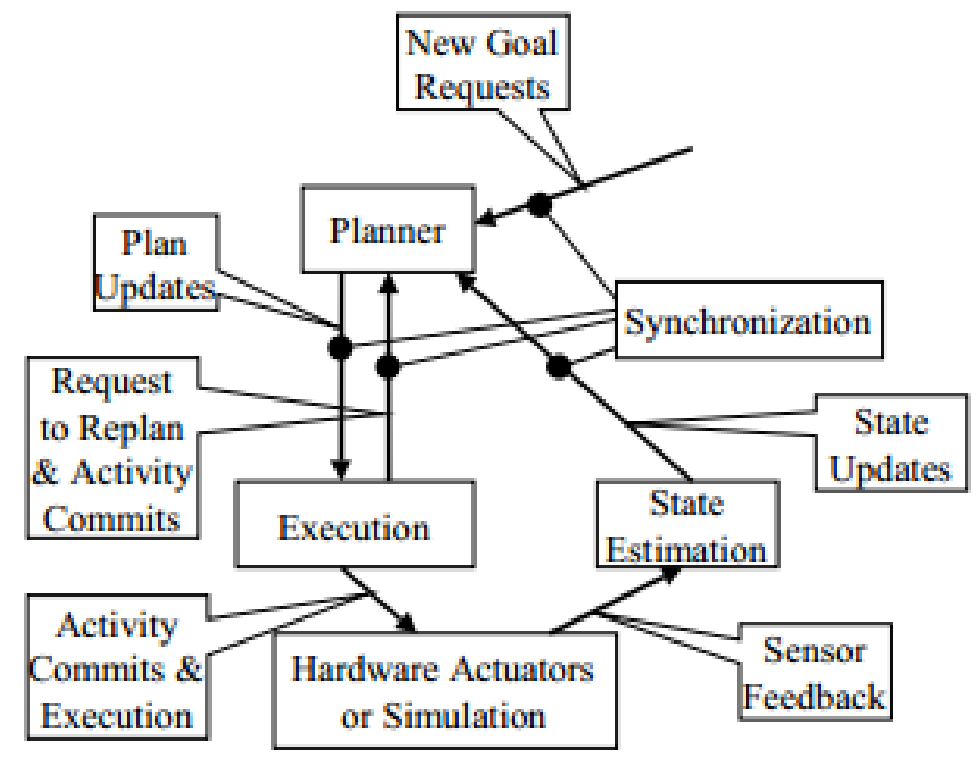

Figure 2.5: The CASPER Architecture (Credit to [11])

\section{Metric Analysis}

Upon examination of the source code [21] of the IPEX CASPER software, the CASPER software is very modular in terms of class structuring and method implementation. As a result of these design decisions, CASPER appears to be easily extensible in the context of scheduling and planning; new features could be added, but any significant changes to the primary focus of the code would more than likely be implemented through a connection to another system (such as ASPEN [25] or the ASE [12]), rather than as part of the CASPER code. Therefore, the CASPER code itself receives a score of Moderate for the Extensibility metric; while CASPER itself could be extended, it is designed in such a way that extending the system through interfacing with other systems is more feasible.

Since CASPER was designed to be deployed on spacecraft, and is currently deployed on a small spacecraft [25] (the target domain), it receives a score of Very Good for the Domain Applicability metric. 


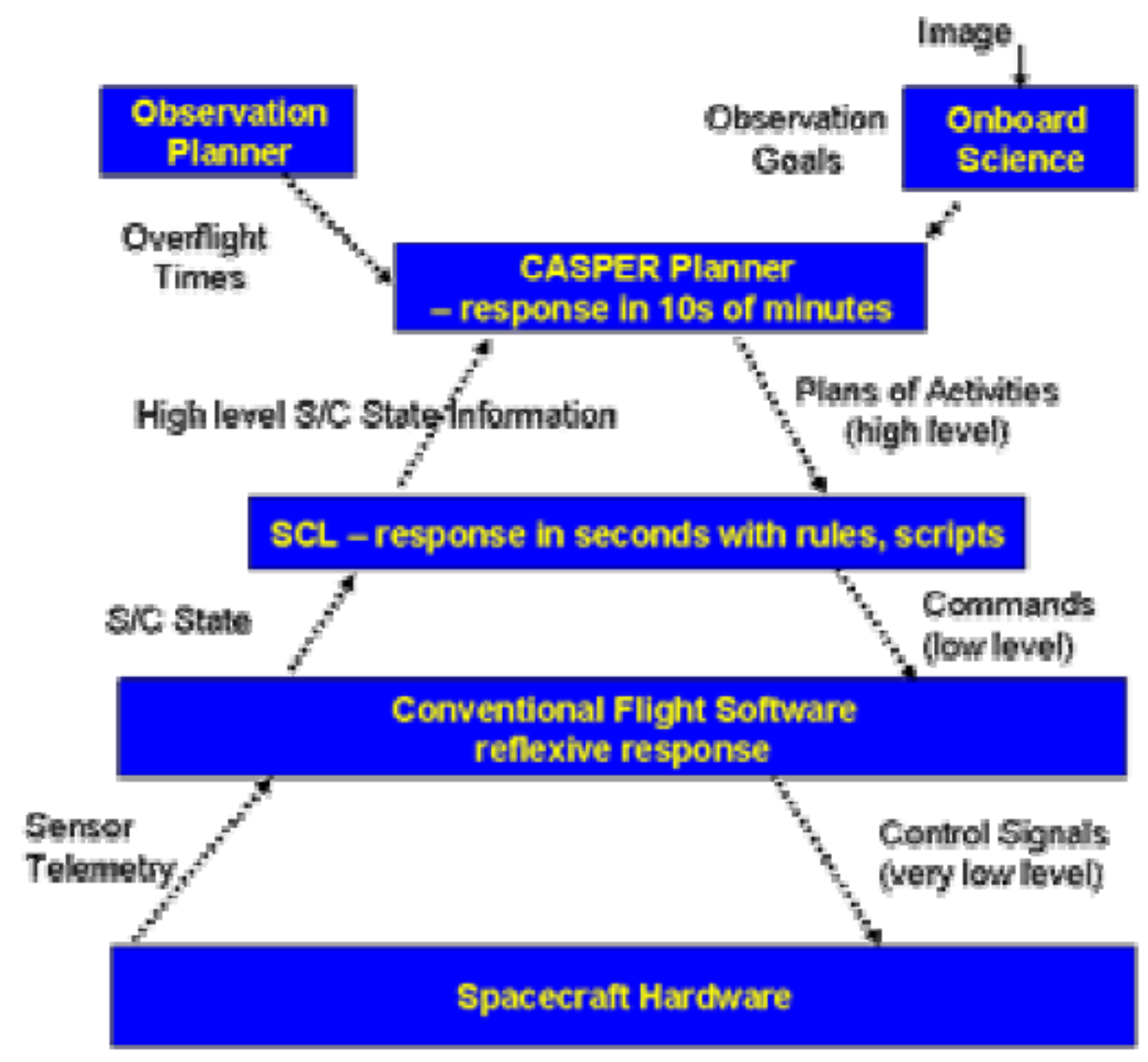

Figure 2.6: The EO-1 Architecture (Credit to [12])

To characterize the performance of CASPER, the developers tested the system on a Sparcstation Ultra 60 with a $359 \mathrm{MHz}$ processor with 1.1GB of RAM; 80 hours of events for the ST4 mission were used as the testing scenario. The average time to generate a correct schedule was 1.134 seconds [11]. Given that current small satellites have much better hardware ${ }^{1}$ [28], the time to generate this schedule would be short enough to have a minimal effect on system execution. Therefore, CASPER is awarded a score of Very Good for the System Overhead metric.

\footnotetext{
${ }^{1}$ i n ter of perfor ran ce; they aren' $\mathrm{t}$ क r rel i abl e क RadHad har dware whi ch typi cal ly have lower har dware speci ficati on $\mathrm{s}$
} 
During the above test, CASPER successfully completed 20 out of 24 activities that it was assigned. While not perfect, CASPER completed an order of magnitude higher number of activities than the batch planning and batch replanning systems [11]. One of the primary shortcomings of the CASPER system is the inability to replan activities outside of activity boundaries; if an extremely long activity fails, CASPER is unable to replan until the activity completes. Additionally, the generated plans may have conflicts and CASPER may not be aware of these issues until it is too late. In light of the shortcomings and successes of CASPER, the system receives a score of Good for the Mission Accuracy metric.

With a full model of the spacecraft and detailed plans of the mission activities, CASPER has decent error recovery capabilities. CASPER is capable of regenerating new schedules or repairing existing schedules to resolve issues with a minimal hit to system performance. However, a major flaw in CASPER's error recovery abilities is that CASPER cannot repair activities that are currently executing; if an activity with an extremely long duration fails, the schedule will only be repaired when the activity completes (on an activity boundary) [11]. Due to the lack of pre-emption, and the potential for error that results, CASPER receives a score of Moderate for the Error Recovery metric.

As with many proprietary, closed-source projects, public documentation of the implementation level details is rare. However, based on the JPL Software Development Process [26], the documentation that does exist undoubtedly would explain all functionality and design in detail to potential users of the system; specifically, the documentation required includes:

- "Design documentation, including functional or architectural design, subsystem specification and detailed design, in-code comments, and imple- 
mentation guidelines, such as design rules, naming conventions, and coding standards."

- "Test documentation, including test plans, test requirements, design of the test environment, test cases, test data descriptions, test procedures, anomaly reports, and test summary status reports."

- "User documentation, including a user's guide, an installation and operations manual, and a release description document, or transfer agreement, that documents as-delivered capabilities, unresolved problems, and recommended work-arounds."

Since the system is already in the target domain, no porting is required. Although there is ample system documentation (assuming the new developer can access it), the system has too much complexity to make it a feasible university CubeSat solution. Configuration requires users that are experts on the spacecraft to use an Agent Communication Language (ACL) to describe the system, and its rules, to the software. This requires the person(s) configuring the system to have an accurate model of the spacecraft, be familiar with the ACL, and be knowledgeable enough to generate accurate rules for the spacecraft. Since CASPER requires a full model of the spacecraft, including an ACL description, CASPER is awarded a Poor score for the Usability metric. Although the documentation for the project is certainly significant, the system also requires an exceptional amount of configuration and has a steep learning curve. Had the target audience of this work been private sector or government development teams with little turnover, the score for this metric would have been higher; however, there simply isn't the time or expertise on a student team for one of them to sift through dense stacks of documentation in an attempt to grasp things they don't have the 
prerequisite understanding for.

Table 2.4: Metric Summary of CASPER

\begin{tabular}{|c|c|}
\hline Metric & Score \\
\hline Extensibility & Moderate \\
\hline Domain Applicability & Very Good \\
\hline System Overhead & Very Good \\
\hline Mission Accuracy & Good \\
\hline Error Recovery & Moderate \\
\hline Usability & Poor \\
\hline
\end{tabular}

\subsubsection{NEAT}

\section{Overview}

The Near-optimal Evolutionary Adaptive Task-manager (NEAT), presented by the Surrey Space Centre at the University of Surrey in $2006^{2}$, is a small satellite system that offers a resource modeller, error correction, and generic scheduling, among other features [9]. Through the usage a of neural network, NEAT tracks resource usage during task execution and corrects its internal model of resources if the usage changes. For example, if a solar panel on a satellite breaks, it limits the rate at which the satellite can recharge its batteries. When the panel breaks, NEAT detects that less power is available to the satellite and changes its internal model to reflect the change; the updated model is then fed into the scheduler during the scheduling process. During the scheduling process, a near-optimal schedule is constructed based on system parameters such as resource usage and

\footnotetext{
${ }^{2}$ the al gori thmwas presen ted in 2005 [ 8]
} 
task priorities. Once the schedule is generated, the first item on the schedule is executed and the rest of the schedule is thrown away. When the executed task completes, the scheduling process begins again and a new task is chosen. This process repeats until there are no more tasks that need to be executed or the system halts. By discarding and regenerating a new schedule every time, the system can be sure that the task it chose to execute was the optimal one.

\section{Metric Analysis}

With most the NEAT system centered around the scheduling algorithm, the modules of the NEAT system appear to be tightly coupled. The resource tracking exists solely to feed back into the scheduling algorithm and all executive functionality is only operational in the context of the scheduler. Because the NEAT system is modular, NEAT receives a score of Poor (instead of Very Poor) for the Extensibility metric.

Like CASPER, NEAT receives a score of Very Good for the Domain Applicability metric; the system was designed for the target domain, deployed in the target domain, and contains many of the same features as GDMFAS.

In the 2005 paper on the NEAT system without a machine learning component, the authors state that NEATs processing demands could be met by dedicating a processor to the system [8]. In a small system such as a CubeSat, where every square centimeter of space counts, it may not be feasible to include

a duplicate processor just to run NEAT. For this reason, NEAT receives a score of Very Poor for the System Overhead metric.

Since each task the system executes is the optimal, or near-optimal, one NEAT receives a score of Very Good for the Mission Accuracy Metric. NEAT's score for 
the Error Recovery metric is heavily influenced by its score for Mission Accuracy; because NEAT automatically schedules the most optimal task to execute next, when a task is terminated due to an error, the best possible choice will replace it. However, while the replacement choice will be the optimal one for the system, it may not resolve the error. This property gives NEAT a score of Good for the Error Recovery metric.

Although NEAT tasks have many of the same properties as GDMFAS, it is unclear how the user should specify these task properties. From the 2005 paper, "user requests for activities are sent to the vehicle interface in whatever format is appropriate for the application in question. The vehicle interface must then translate these into standardised operation requests before passing them on to NEAT". Both the 2005 and 2006 NEAT works require an intermediate interface that is specific to the mission; some software interface must exist to translate user formatted commands to something NEAT can operate on. While this requirement isn't terribly different from GDMFAS's requirement for tasks to be outlined in a configuration file, no additional software needs to be developed for GDMFAS's configuration. Where NEAT does succeed in Usability is the relatively straightforward design. Although NEAT uses genetic algorithms [8] and neural networks [9], which may not be familiar to student software developers, they aren't terribly difficult for students to learn; the modular design (despite it being highly-coupled) is a strong point, but decent documentation on system implementation details is notably absent. As a result of the additional software interface development required by NEAT, the system receives a score of Moderate for the Usability metric. 
Table 2.5: Metric Summary of NEAT

\begin{tabular}{|c|c|}
\hline Metric & Score \\
\hline Extensibility & Poor \\
\hline Domain Applicability & Very Good \\
\hline System Overhead & Very Poor \\
\hline Mission Accuracy & Very Good \\
\hline Error Recovery & Good \\
\hline Usability & Moderate \\
\hline
\end{tabular}

\subsubsection{Other Notable Systems}

In addition to systems reviewed in this chapter, other notable systems include NASA's New Millennium Remote Agent Architecture (NMRA) [32] [15], NASA JPL's ASPEN [39], and APL's SciBox [13].

\subsection{Literature Summary}

A table containing metric scores for all reviewed systems is presented below in Table 2.6. 
Table 2.6: Literature Metric Summary

\begin{tabular}{|c|c|c|c|c|c|}
\hline Metric & CFS & LOGOS & ASOF & CASPER & NEAT \\
\hline Extensibility & Good & Very Good & Very Good & Moderate & Poor \\
\hline Domain Applicability & Moderate & Good & Moderate & Very Good & Very Good \\
\hline System Overhead & Very Good & Moderate & Good & Very Good & Very Poor \\
\hline Mission Accuracy & Very Poor & Good & Very Good & Good & Very Good \\
\hline Error Recovery & Poor & Poor & Good & Moderate & Good \\
\hline Usability & Very Good & Very Poor & Moderate & Poor & Moderate \\
\hline
\end{tabular}




\section{Chapter 3}

\section{GDMFAS Design}

This chapter discusses the overall design of GDMFAS and its components. Much of this design is at a high level, although some of the discussion borders on implementation details. Information on the actual implementation can be found in Chapter 4.

In order to facilitate the quick development of small autonomous systems, the Generic Decision Making Framework for Autonomous Systems was developed. Unlike many previous works, GDMFAS was designed with a focus on a small code base, ease of use, and extensibility. GDMFAS is made up of three components: the System Monitor, the Scheduler, and the Task Manager. At a high level, the System Monitor is responsible for monitoring the resources of the system and handling failures. The Scheduler is in charge of putting together a sequence of Tasks that the System Monitor executes. The Task Manager keeps track of all Tasks in the system and provides an interface for the Scheduler to pull Tasks from the Task Database. There are multiple different tiers of Tasks the Task Manager keeps track of, and each has its own data items associated with it; the different tiers of Tasks (schedulable units), and the relevant data items are 
outlined in the following section.

\subsection{Definition of Tasks and Data Items}

In GDMFAS, a Task is the smallest unit that can accomplish something and be placed on a schedule. Each task is comprised of the data shown in Table 3.1.

As we can see from Table 3.1, each Task has a set of resources that it requires to execute. These resources can either be depletable, non-depletable, or static. This work defines depletable as a resource that is used and never replenished (such as something deployed by the system), while non-depletable is defined as a resource that is used but can be replenished (i.e using disk space to write a file and then deleting the file), and static is defined as something which cannot decrease in capacity (such as a camera). In addition to a resource requisite, each Task also has a priority given to it by the user. This priority is used by the system to decide where to place this task relative to other tasks when scheduling.

However, just because a certain Task has the highest priority doesn't mean it will execute first. If the Task with the highest priority is very dangerous (low chance of success) and is not time sensitive, another task may be placed before it by the Scheduler. Every Task also has an Estimated Time to Completion (henceforth ETC) which is the developers estimate of how long this particular Task will take to execute. This estimate should be based on a combination of the developer's experience with the system and the results of testing. The unique identifier is simply a tag that allows the Task to be uniquely indexed into the Task Manager's data structure. The prerequisites for a Task is where the structure gets a bit more complicated. On the surface, it seems that the prerequisites are just those contained within the array. However, since all Tasks 
Table 3.1: Properties of a Task

\begin{tabular}{|c|c|c|}
\hline Name & Type & Description \\
\hline Task Name & String & $\begin{array}{l}\text { The human readable identifier } \\
\text { for this task (optional) }\end{array}$ \\
\hline Resource List & TaskResource[ ] & $\begin{array}{l}\text { The resources, and their quan- } \\
\text { tities, that this task requires }\end{array}$ \\
\hline Task Priority & int & $\begin{array}{l}\text { The user-assigned priority of } \\
\text { this task. }\end{array}$ \\
\hline Estimated Time to Completion & int & How long the task takes \\
\hline Unique Identifier & int & $\begin{array}{l}\text { A unique number representing } \\
\text { this task }\end{array}$ \\
\hline Prerequisites & Task[ ] & $\begin{array}{l}\text { The collection of tasks that } \\
\text { must be completed before this } \\
\text { one }\end{array}$ \\
\hline Longest Chain & int & $\begin{array}{l}\text { The length of the longest chain } \\
\text { of prerequisites which are not } \\
\text { complete }\end{array}$ \\
\hline Completed & boolean & $\begin{array}{l}\text { Whether or not this task is } \\
\text { done. Repeatable tasks can } \\
\text { never be marked completed }\end{array}$ \\
\hline Probability of Success & byte & $\begin{array}{l}\text { A value between } 0 \text { and } 100 \\
\text { where } 0 \text { is certain failure and } \\
100 \text { is certain success }\end{array}$ \\
\hline
\end{tabular}


have this field as well, there can be a number of very large dependency trees for this Task. For this reason, each Task also stores the length of the deepest tree of prerequisites. Finally, each task stores its completion state. For Tasks which should be repeated, the value of the Completed field constantly remains set to false.

Given the definition of a Task, it also critical to understand how a Resource is defined in the context of this framework. In the context of GDMFAS, a Resource is essentially a tool that the system uses to complete a task. For example, if the Task were to take a picture of the Earth some of the Resources needed would be a camera to take the picture, disk space to store the picture, and power to run the camera and keep the autonomous system running. In this example, the camera is a static resource, while the disk space and power are non-depletable, assuming the autonomous system is equipped with solar panels. The underlying data for an Abstract Resource is shown in Table 3.2. When viewing the table, note that AbstractResource is the parent class of both Resource and TaskResource.

\section{Table 3.2: Properties of an Abstract Resource}

\begin{tabular}{|l|l|l|l|}
\hline Name & Type & Description & Specific To \\
\hline Name & String & $\begin{array}{l}\text { The human readable identifier for this } \\
\text { Resource (ex: power) }\end{array}$ & ALL \\
\hline Shared & boolean & $\begin{array}{l}\text { Whether or not two Tasks can share } \\
\text { this Abstract Resource }\end{array}$ & Resource \\
\hline Amount & int & The amount the resource is used & TaskResource \\
\hline
\end{tabular}

When implemented in the framework, an Abstract Resource can take on two forms. The first form, a Resource resides in the System Monitor and is used to keep track of all resources available within the system. This Resource 
is used to make decisions based on what the resource levels are. On the other hand, a TaskResource is used to predict to what degree executing a given Task will affect overall system resources (Resources). Thus, a TaskResource is used to predict what the resource levels will be in the future.

Schedulable units, like Resources, also have a hierarchy. Tasks have already been explained and are the most common schedulable unit in the system. Activities are the mid-level building blocks and are composed of Tasks. The highest level building block is called a Mission Objective and is composed of Activities. The GDMFAS system does not automatically create Activities and Mission Objectives from Tasks. In order to be as accommodating as possible, the classification of what constitutes a Activity and Mission Objective has been left up to the developer of the system. Because all schedulable units are derived from a common abstract building block called a SchedulableItem, Activities and Mission Objectives can be used interchangeably with Tasks in any listings that require a SchedulableItem (for example, any prerequisites). This interchangeability allows the developer more control over the expression of the goals of their mission and other needs. If the developer would like the prerequisites for a Task to be only other Tasks, then that is acceptable. On the other hand, the developer can also make sure that a Task will not execute until a Mission Objective has been completed. This versatility allows the developer fine-grained control during the planning process and ensures that the system will execute SchedulableItems in a manner consistent with the way the developer wants the system to. 


\subsection{Task Manager}

This section first presents an overview of the Task Manager and then explains the parts of the design in more detail.

\subsubsection{Task Manager Overview}

The Task Manager is the module that controls the database of all possible Tasks the system can execute. In addition to storing Tasks, the Task Manager is also responsible for adding new Tasks to the database, updating the Task's properties, keeping track of what Tasks have been completed (and thus mission progress), and relaying Task information back to the humans in charge of the system.

\subsubsection{Adding a New Task}

There are two methods for adding a new Task into the system. The first method is adding the Task at system creation time. When the system is created, the developers define what the initial set of Tasks are. Although this method is the most straightforward, it is also the most limited. Tasks cannot be added or modified using this first method. These limitations are alleviated by allowing the system to receieve Tasks from an external source. In the context of a spacebased autonomous system, this external source would be the earth station for the mission. Once a Task is received from the external source (hereafter referred to as "the ground") the Task is first processed, and then added to the Task Repository or the Ready Pool. 


\section{Processing}

During the processing stage, a Task is evaluated to determine if it has any dependencies. If there are dependencies, these dependencies are then checked for completion. If all dependencies are complete, the Task is then added to the Ready Pool, otherwise the Task is added to the Task Repository.

\section{Ready Pool}

The Ready Pool is essentially the same thing as the Task Repository except that it houses only those Tasks which are ready to be scheduled (those with all prerequisites complete). Although the qualified Tasks are never physically stored in the Ready Pool, the Ready Pool keeps a pointer to them to conserve memory.

\subsubsection{Update of Task Properties}

When a task is executed by the System Monitor (discussed in Section 3.4), the actual usage of non-static Resources is logged by the System Monitor. The actual values for a particular execution are then put together with the previously estimated Resource usage and a new value is calculated. This new predicted value for each resource is then sent from the System Monitor to the Task Manager and is assigned to the Amount field of each TaskResource used by the recently executed Task. Static TaskResources are not modeled since they never change. 


\subsubsection{Relaying Task Status}

In addition to scheduling and completing Tasks, GDMFAS also has the ability to report the status of the mission to the ground. Although the exact format of the mission status report is configurable at system creation time, the report typically contains and overview of all Mission Objectives, Activities, and Tasks that have been completed. By default, the overview is formatted such that the set of all reported Tasks $\not \subset$ Activitys and the set of all reported Activitys $\not \subset$ Mission Objectives. Additionally, the default configuration reports the overall mission progress using Equation 3.1.

$$
\begin{aligned}
& \text { Mission Progress }=\frac{\text { MissionObjectivesComplete }}{\text { TotalMissionObjectives }}+ \\
& \frac{\text { ActivitiesComplete }}{\text { TotalActivities }}+\frac{\text { TasksComplete }}{\text { TotalTasks }}
\end{aligned}
$$

In addition to reporting the numerical progress of the mission, lists of completed SchedulableItem are also communicated to the system operators. There are separate lists for Mission Objectives, Activities, and Tasks, each with their own completion percentage reported. An example completion report is shown below (... is used for brevity, but in practice everything will be listed to the operator):

Mission Completion: $44.047 \%$

Mission Objectives Complete: 1/3 (33.333\%)

1) Collect environmental data on the surface of Mars and relay it to the ground

Activities Complete: 4/14 (28.571\%) 
1) Collect data

4) ...

Tasks Complete: 13/56 (23.214\%)

1) Log temperature data

2) Log air pressure

$\cdots$

13) Take photo

Due to the limited nature of many small satellite communication links (typically no more than 9600bps [20] [24]), this progress data is not transmitted as is, but rather sent in a binary format which includes the relevant data the receiver would need to construct the text report from the data (Task IDs for mapping names to ID numbers, et cetera) into a binary format and then transmitted. The binary data is then decoded when it arrives at the receiver, resulting in a lower transmission overhead and less computations on the autonomous system.

\subsection{Scheduler}

The Scheduler is the module that consolidates all of the analysis data from the Decision Making Modules (DMMs) and constructs a schedule. While the scheduler could be adapted to provide finer detail, if it important to note that the scheduler is not intended to be a hard real-time scheduler. Since the granularity required by past and current Cal Poly CubeSats has never been real-time, this is acceptable for the target domain.

The DMMs housed in the Scheduler are the Risk Manager and the Prioritizer. 
For the purposes of the design in this section, the implementation details of the Scheduler such as the implementation language, algorithm complexity, et cetera are omitted. Instead, the Scheduler is discussed here at a high level. In order to construct a schedule, the Scheduler first invokes each DMM with a given Task as a parameter. After being passed through the DMMs, the modified list of Tasks is used to construct a schedule. The scheduling life cycle is shown in Figure 3.1.

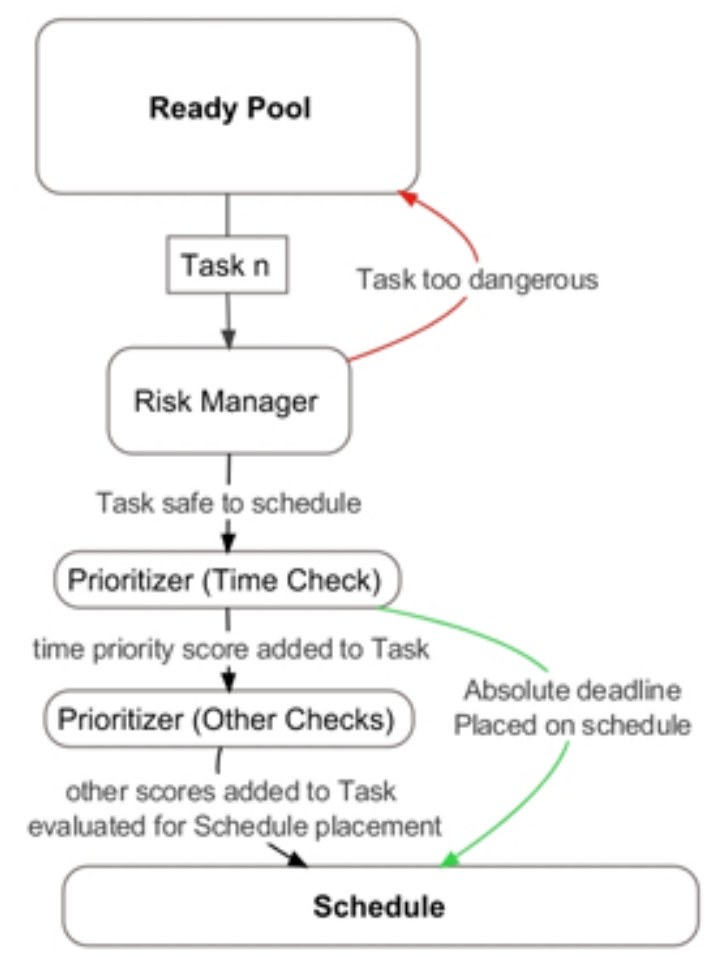

Figure 3.1: The scheduling process

As the Task passes through the process shown above, each module analyzes the Task. If any DMM decides that the Task is a bad candidate, the pipeline terminates and the Scheduler repeats the pipeline with the next Task. A more detailed explanation of the selection process is given after an overview of the DMMs. 
Once enough good candidates are found to make a schedule, the Scheduler stops the pipeline and constructs a schedule. The completed schedule is then passed back to the System Monitor. A more detailed look at the DMMs is presented in the following subsections.

\subsubsection{Risk Manager}

The Risk Manager, as its namesake implies, evaluates how risky executing a specific Task may be. Unlike the Prioritizer, the Risk Manager does not rank Tasks. Instead, the Risk Manager removes Tasks from the selection pool. The decision to remove a Task from the item pool is based on a number of criteria. The first criterion is the probability of success. The initial probability of success is supplied by the developer and can be updated later through remote commands. As Tasks execute, the GDMFAS system will update the probability of success if a success evaluation function is supplied. If a Task has a probability of success below the user-supplied threshold, the consequences of failure are then examined. Consequences are examined using a user-supplied risk evaluation function, which returns the degree of risk a particular consequence entails, or the default evaluation function. The default evaluation function uses a number of factors to make its decision and is only invoked in the absence of a user-defined risk evaluation function. The factors examined by the default function are probability of success, the resources used by the Task, the user priority of the Task, and the current status of the mission. If the probability of success is below the user-defined threshold (or under $65 \%$ if there is no supplied threshold) the current status of mission will be evaluated using the user-specified progress evaluation function, or the default one in Equation 3.1. If the mission is far enough along, defined by a user-supplied mission state evaluation function (or is its absence the default value of $70 \%$ ), then 
the low success Task will be passed through to the Prioritizer. If the mission is not far enough along, the low success Task is removed from the selection pool. If the Task is not removed through the success rate and mission status filters, the resources the Task uses are examined next. If the Task uses an amount of resources that is greater than the amount remaining or the amount will put the system in an unsafe state (i.e low power), the Task is removed from the selection pool. The dangerous Tasks removed from the selection pool will only be executed when explicitly remotely commanded or the state of the system changes. The latter option is discussed further in Section 3.4. It is important to note that if the Task has a low probability of success, but is not deemed dangerous, the Task will not be removed from the selection pool. However, the low probability of success will be noted when the Task is passed through to the Prioritizer.

\subsubsection{Prioritizer}

The Prioritizer is the final DMM in the Task evaluation control flow and assigns a ranking to each Task. This ranking is used by the Scheduler to construct a schedule. The ranking assigned by the Prioritizer is calculated using a number of factors.

\section{Time}

The first factor taken into account is the time sensitivity of a Task. In the context of the GDMFAS system, there are two types of time constraints. The first type of time constraint is called a situational time constraint. This type of constraint is applicable to Tasks which require specific times of the day to execute. An example of a Task with this type of constraint would be sampling 
the daytime temperature on a planet. The example Task would receive a higher ranking in the day time and a much lower ranking (enough to prevent it from getting scheduled) at night. Despite the ability to make decisions involving time, The GDMFAS does not have actual knowledge of the time. This information must be provided to the system in the form of a user-defined time function. The other type of time constraint is called an absolute deadline. If a Task has this type of constraint placed on it, it must be executed by the system by that time (either absolute time or time relative to the start of the mission). Because of the nature of this type of constraint, any Task that has this type of constraint is immediately returned to the Scheduler without any further processing by the Prioritizer. The reason for this is to ensure the Task gets executed in time, but details of this are discussed in the algorithm subsection.

\section{Fairness}

The second factor the Prioritizer takes into account is fairness. Due to the large number of Task properties to account for when choosing a Task, it is very possible that a Task with very unfavorable traits (no time constraints, moderate probability of success, et cetera) will never be scheduled to run and become starved. To prevent this, the Prioritizer assigns a higher ranking to tasks which have not be allowed to execute recently. The longer the task has been starved, the higher the boost in the rankings. Because potentially dangerous Tasks have been filtered out of the selection pool by the Risk Manager, Tasks which have never run due to danger will not be considered for a boosted ranking due to starvation. If a Task has been starved because its prerequisites have not been met, its prerequisites will receive a ranking boost in an attempt to increase the Tasks chances of executing in the future. 


\section{Contribution to the Mission}

The third factor the Prioritizer takes into account is the potential effects on the mission that will result from completing a Task. If the completion of the Task being considered will also complete a Activity (especially a Activity which will then complete a Mission Objective), it will receive a boost in its ranking. Additionally, if the Task is a prerequisite of a large number of other Tasks it will receive a boost in its ranking. The more Tasks the Task is a prerequisite for, the higher the boost.

\section{User Priority}

The final factor the Prioritizer considers is the user-assigned priority. The user-assigned priority is simply a constant value that gets added to the ranking of a Task. This value takes the form of a Double and is limited to values between 0 and 10,000 inclusive. The higher the user priority, the more important a Task is relative to other Tasks and the more important it is to the developer that a Task gets executed.

\subsubsection{Scheduling Algorithm Overview}

The first step in the scheduling process is to place all Tasks with absolute time constraints on the schedule. This step ensures that no Task will miss its deadline. The next step in the scheduling process is to place the Tasks from the selection pool on the schedule. The Tasks are placed on the schedule in ranked order with one exception. If there is a space on the schedule in which the next Task being considered cannot fit, then the scheduling algorithm looks through the selection pool for the highest ranked Task that will fit in that time slot. This 
situation will only occur if there are Tasks with absolute deadlines already on the schedule. The scheduling algorithm ceases execution when a schedule of the specified length is constructed. This length is specified by the developer and can be increased to reduce usage of system resources to create schedules or the length can be decreased to increase the adaptivity of the schedule. After the schedule has been constructed, it is passed to the System Monitor and the Scheduler ceases execution until invoked again by the System Monitor. A control flow diagram of the scheduling algorithm is shown in Figure 3.2 and pseudo-code for the main control loop is shown in Algorithm 1. Pseudo-code for placing a Task on the schedule is shown in Algorithm 2.

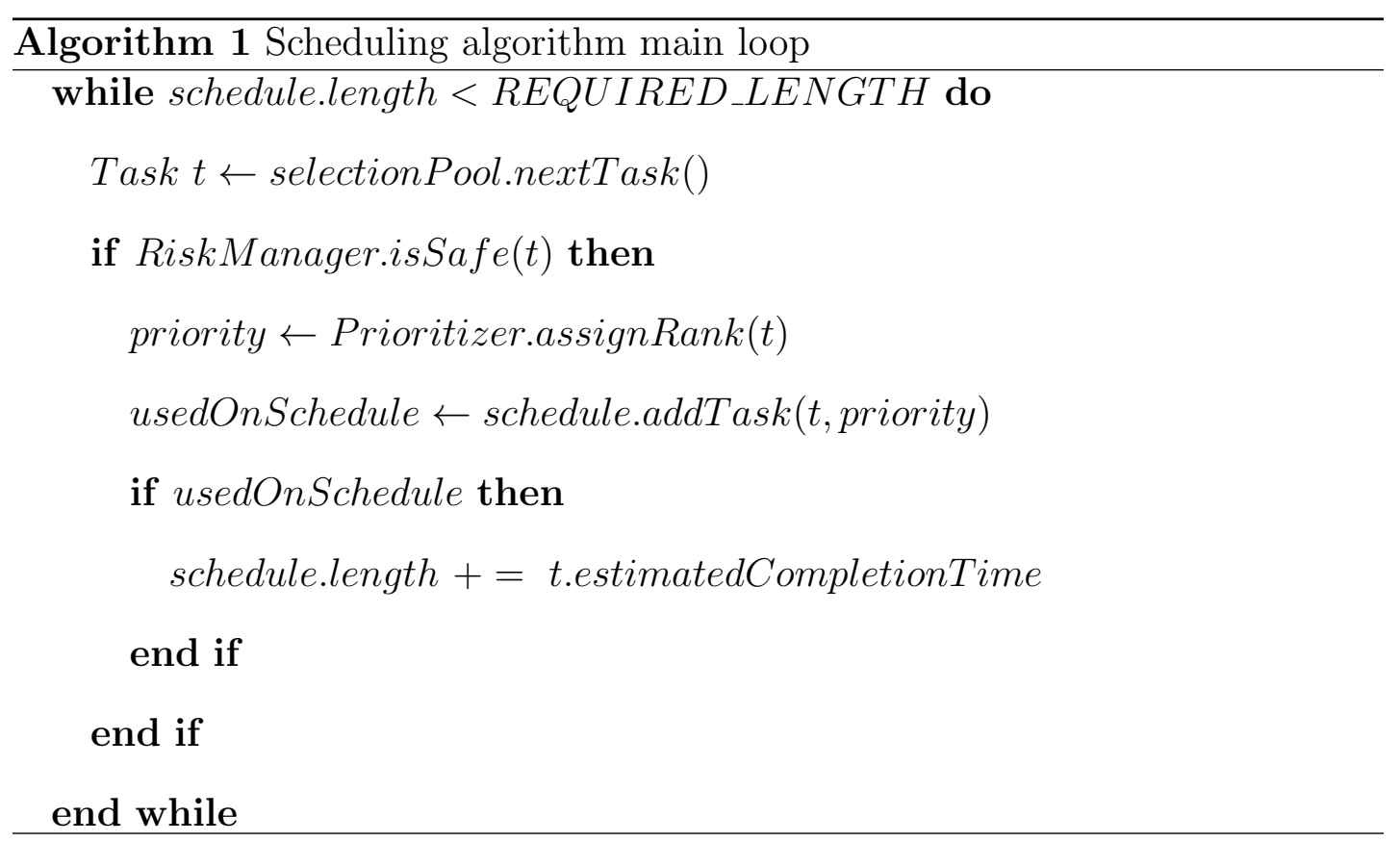

Figure 3.2: A visual representation of the addTask function. Note that Tasks with absolute deadlines must begin by their deadlines. Although Task 3 had a higher priority than Task 1, it did not fit in its place. As a result, it is placed after the Task with an absolute deadline. 


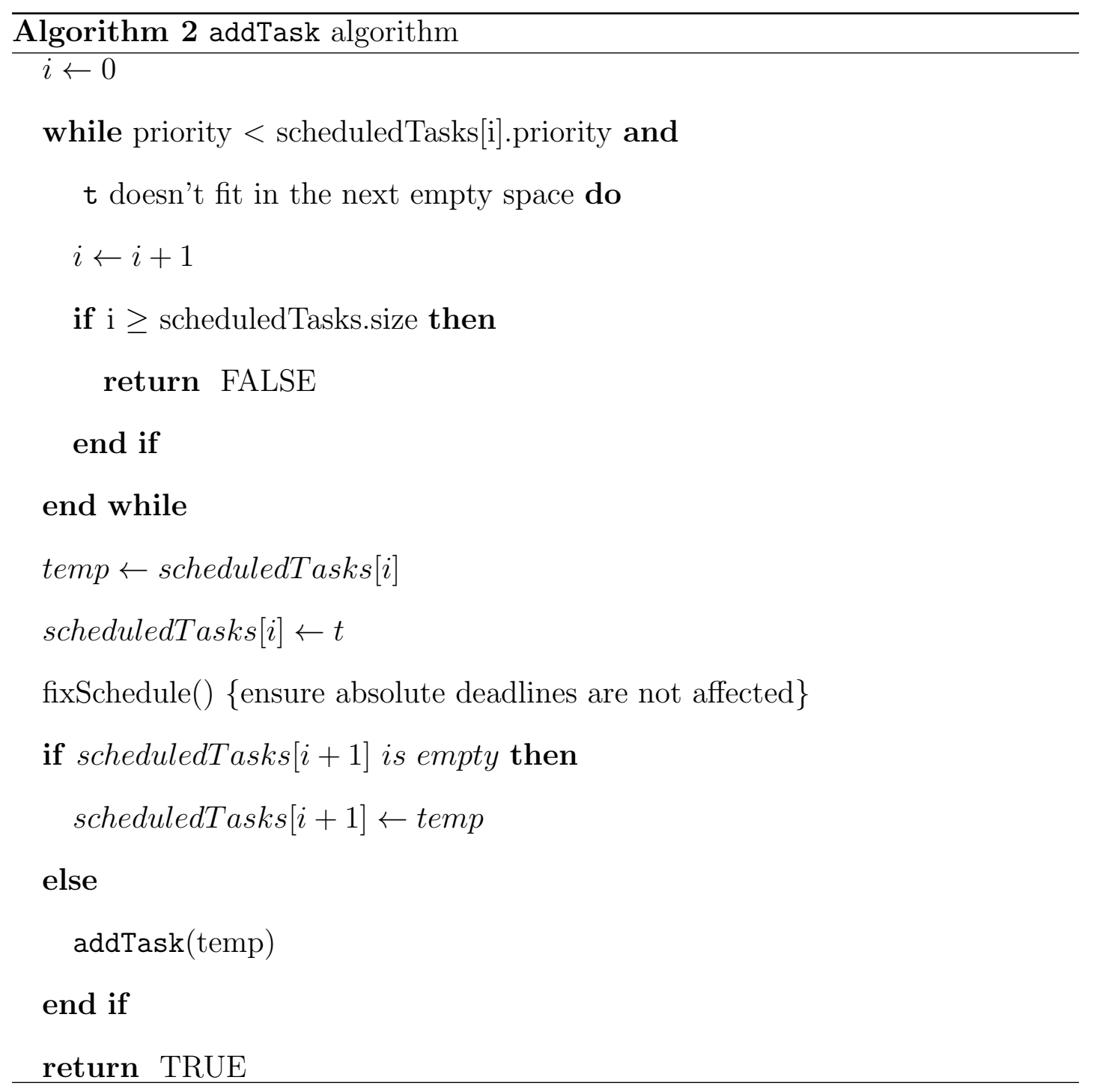




\subsection{System Monitor}

The System Monitor (SM) acts as the top-level module, or brain, of the framework. At any given point in time, the SM can invoke the Scheduler or the Task Manager. In addition to invoking other modules. the System Monitor is also responsible for overall system safety. The System Monitor also monitors system resources, the system alert level, and the schedule. Finally, the System Monitor is also responsible for processing data coming in from the Communication Module.

\subsubsection{System Safety}

As the top level module, it is the responsibility of the System Monitor to make sure that everything goes according to plan. This is accomplished through the use of resource modelling, monitored execution of Tasks, enforcing the mandatory execution of critical system Tasks, and ensuring that human intervention is possible at any time.

\subsubsection{Resource Modelling}

In the GDMFAS system, there are two resource modelling systems provided. The two resource modelling systems have different complexity and should be chosen at system compile time for the purposes of reducing code size. The first resource modelling system RMS is a neural network. As in [9], the inputs to the network are the estimations of the resource usage, while the outputs are the corrected resource values. These corrected values are then used as future estimations of resource usage for scheduling and planning of the Task. The other option provided (averaging) is much simpler, but may be less accurate. In this second method, 
the previous usage levels are averaged together with the most recent usage level. While this method is crude, is has very low overhead and offers at least some correction to the estimated resource use.

\subsubsection{Monitored Execution and the System Alert Level}

When a Task is executed by the System Monitor, it is monitored by the System Monitor throughout its life cycle. Because the assumption that the GDMFAS system is the only one running on the autonomous system is a poor one, the System Monitor checks Resource levels before the execution of Tasks to make sure the required amount of resources are available and have not been modified externally. As the Task executes, the System Monitor checks the system resource levels and will suspend or kill the Task at any sign of danger (ex: critically low power levels). In addition to resource usage problems, a Task will also be pre-empted if it takes significantly longer to execute than expected. If "longer than expected" is not defined by the user, the default maximum of twice the expected time is used. Although the default method of dealing with a troublesome Task is to halt or kill them, the System Monitor may take other steps depending on the System Alert Level (SAL). By default, there are only three SALs but the user can define more levels or replace the current SALs entirely. The three default SALs are "Safe", "Warning", and "Danger".

\section{Safe}

"Safe" is the default state at initialization of the GDMFAS and is an indicator of a health system with acceptable resource levels. 


\section{Warning}

The "Safe" state will change to "Warning" under two scenarios. The first scenario is if something has gone wrong but has no system crippling effects (i.e an experiment payload has malfunctioned). The other case in which the state will change to "Warning" is if there are low Resource levels (critical resources such as power below an acceptable level). Upon transitioning to the "Warning" state, the System Monitor will be more strict when judging if Tasks are allowed to run. For example, if there is only $30 \%$ power remaining then a Task that requires $20 \%$ power to run may be prevented from executed. This prevention occurs because the Task's $20 \%$ power requirement is simply an estimation and may actually turn out to be $30 \%$. Even though the Monitored Execution will catch this problem while the Task executes, it is the opinion of the author that the number of actions which degrade system state should be minimized. Unless otherwise overridden by developers, the SAL will only return to "Safe" from "Warning" if the Resource levels return to acceptable and current "Warning" state was a result of low Resource levels.

\section{Danger}

The state will only change from "Safe" to "Danger" if there is something fundamentally wrong with the system (i.e there is no longer any way to read power levels). If this transition occurs, then by default it will never change to any other state unless this fundamental problem fixes itself (i.e power queries begin to respond with reasonable values). Transitions from "Warning" to "Danger" resulting from critically low Resource levels can be reversed if the Resource levels rise above the critical level. Upon transitioning to the "Danger" state, the System 
Monitor will only execute recovery tasks (if they exist), critical system tasks (such as replenishing power), and the most trivial of Tasks. If the system is placed into the "Danger" state as a result of anything other than low Resource levels, the System Monitor will try to transmit a status message containing diagnostic information at a user-specified rate using the Communication Module. Even if the "beaconing" has been disabled by the user (either at compile time or by sending a command) the operators on the ground can still manually request this information from the system.

\subsubsection{Scheduler Override}

As discussed in Section Scheduler, the Scheduler creates a schedule and then passes it back to the System Monitor. By this time, the schedule will already contain a number of items that have been selected. Before executing a Task (or after pre-empting one) the System Monitor has other choices it can make besides executing the Task on the schedule. The first alternative is to execute a critical system Task (such as ground communication) instead. This may be necessary if the ranking assigned to a system Task in the scheduling algorithm is consistently low. The other alternative to executing a scheduled Task is to execute a Task that has been sent to the system from the ground. If any Task is executed instead of a scheduled Task the Scheduler two things may occur. If the Task was less than or equal to the duration of the scheduled Task, then the System Monitor can continue executing the schedule as if the Task from the schedule had been executed. Alternatively, the Scheduler can be invoked again to make a new schedule that accounts for the change in execution. 


\subsubsection{Communication Module}

The Communication Module or CM, is a module that represents the communication device and operations in the system. The primary function of this module is to receive and process commands from the ground and to send data to the ground. This module is the conduit through which new Tasks and configurations are introduced into the system after compile time. Before sending data down to the ground, this module is responsible for any processing (such as compression) that occurs. If commanded, this module also has the ability to manage the entire GDMFAS system. For example, the CM is able to restart the System Monitor or kill any other module. Essentially, if the System Monitor can do something in the system, the CM can be commanded from the ground to do it as well. This design decision was made to ensure that the system is also able to be overridden by humans, if necessary. 


\section{Chapter 4}

\section{Implementation}

This chapter gives an overview of the GDMFAS implementation and provides finer detail for an example implementation using GDMFAS.

\subsection{Framework Implementation}

\subsubsection{Overview}

GDMFAS is implemented as a collection of classes in $\mathrm{C}++$. Most modules that have been listed in the design as distinct units are also present as classes. Wherever possible, the control classes make use of polymorphism to ensure the maximum level of extensibility. As in the design, each of the three major components is a distinct unit and has been implemented as its own process. These processes communicate with each other through the use of sockets. Specific implementation

details for each component are provided in the following subsections. Note that some classes, such as the Scheduler, have not been fully implemented and do not reflect the design specifications due to time constraints. 


\subsubsection{Resource Implementation}

The Resource class is the parent class of any resources that are tracked in the system. The class has the following virtual methods that are called by the System Monitor and should be implemented by all derived classes:

- int updateValue()

- $\operatorname{char} \operatorname{attach}()$

- char detach()

\section{int updateValue()}

This method is called by the System Monitor to update the current value of the Resource. The method is the only interface for updating resource values; therefore, any methods required to update the value (and return it) must be callable via this method. Like NEAT [9], GDMFAS requires that all resource values are scaled to a value in a specified range. The scaled value must be between 0 and 1000. NEAT specified a range of 0.0 to 1.0, but GDMFAS uses 0 to 1000 to decrease the number of floating point computations required by the system; some, if not most, embedded architectures do not have hardware support for floating point operations, forcing the software to emulate the FPU. For static resources, a value greater than 1500 should be returned (giving a margin of error if more of the resource that the anticipated maximum exists). This value frees up negative values (such as -1 for consistency) to be used as error codes. 


\section{$\operatorname{char} \operatorname{attach}()$}

This method is called by the System Monitor when it wants to start monitoring this Resource. Any methods that would normally be callable from an init () method should be called from this method. Errors in the initialization are reported as -1 , while success returns 0 .

\section{$\operatorname{char} \operatorname{detach}()$}

This method is called by the System Monitor when it wants to stop monitoring this Resource. detach can be considered the opposite of attach and should generally undo any actions performed in attach. As with the attach method, it should return 0 on success and -1 on failure.

If the user of the framework decides not to override these methods, a default value that has no effect on the system is provided; attach and detach return a successful initialization/cleanup and updateValue returns a value greater than 1500 (indicating a static resource that isn't tracked).

\subsubsection{Task Implementation}

Tasks are implemented in a class that is based on a process model. It includes methods such as bool isActive(), char terminate(), and char start().

\section{Task Execution}

Tasks are executed using the start() method, which utilizes the fork() and exec() paradigm. This provides the stability of pre-existing Linux process functionality and is a familiar interface to the users of the system. It also provides 
the process ID to the System Monitor and allows the System Monitor to easily stop, resume, and terminate the process.

\section{Task Termination}

Task termination is implemented through the terminate,die, and killWait methods. terminate sends the SIGTERM signal to the process, allowing it to perform clean up actions and gracefully terminate. If the process doesn't terminate after a SIGTERM, die sends the SIGKILL signal, gracelessly killing the process. In both cases, the killWait method is called to wait for the process to terminate, although there is likely to be very little delay in a process terminating from a SIGKILL.

\subsubsection{Component Communication Implementation}

As previously mentioned, the three primary components communicate with each other via datagram (UDP) sockets. For each component, there is a set of command packets defined. Example commands include the Scheduler requesting the Ready Pool from the Task Manager in order to perform its scheduling algorithm and the System Monitor updating the status (completion, resource usage, et cetera) of a Task in the Task Manager.

Although the three primary components reside on the autonomous system in the default configuration, the use of UDP/IP instead of file-based UNIX sockets allows components of the system to be easily moved elsewhere. The use of UDP/IP also allows new components both on and off the spacecraft to be easily integrated into GDMFAS. For example, a ground-based data collection system, such as Cal Poly's Massive Operations, Recording, and Experimentation Database Sys- 
tem (More dBs) [33], could interface directly with the spacecraft; however, this link must operate on top of radio-specific layers such as those provided by the ION delay tolerant network (DTN) [18].

\subsubsection{Task Manager Implementation}

In the prototype implementation, the Task Manager includes methods for adding, removing, and retrieving Tasks. Tasks are stored internally in the Task Repository using the standard $\mathrm{C}++$ list data structure, since accessing any element in the list is a $\mathbf{O}(\mathbf{N})$ operation, and inserting and deleting items anywhere in the list is a $\mathbf{O}(\mathbf{1})$ operation. The structure is also particularly conducive to sorting algorithms [2]. To save on memory and reduce complexity, the Ready Pool is accessed through a method that builds the Ready Pool on demand; this method has $\mathbf{O}(\mathbf{N})$ operation. At start-up, Task data is read from a configuration file and the corresponding Tasks are constructed and added to the Task Repository. In the prototype implementation, the Task Manager reads a canned set of Tasks from the configuration file.

\subsubsection{Scheduler Implementation}

In the prototype implementation, the Scheduler includes methods for generating a schedule using the basic scheduling algorithm and the two provided DMMs discussed in the design. These two classes, the Prioritizer and the Risk Manager, are both derived from the DMM parent class. The DMM base class only has one virtual method with the signature list<Task $*>$ evaluate (list<Task $*>$ ). At the end of the DMM iteration, the result is the list of all Tasks that should be scheduled in order of priority. Due to the generic implementation, a virtually 
unlimited number of DMMs can act as Task filters during the scheduling process and the resulting schedule can be as simple or as complex as the user wants.

\subsubsection{System Monitor Implementation}

In the prototype implementation, the System Monitor is the most complete of the three modules. The class contains a list of Resources that represent all the resources in the system and a list of TaskResources that represent all the resources the currently executing Task utilizes. All of the process spawning and termination is encapsulated in the previously discussed Task class, so the System Monitor is completely agnostic about the majority of the low-level details in the system. The System Monitor sits at the core of the system controlling everything through method calls to other classes. For example, if a Task misbehaves the System Monitor terminates the Task; however, the actual system calls to kill and fork are encapsulated in the Task class. Once a Task is spawned, the System Monitor retrieves the process ID of the Task, calls the attach() method of all of its resources to begin the tracking process, and monitors the Task's execution.

Although low-level mechanisms for process interaction are encapsulated in the Task class, the System Monitor is ultimately responsible using these mechanisms via the Task API. An example of this is process termination. In GDMFAS this is

a two stage process. First, the Task's process ID (PID) is sent a SIGTERM to give it the opportunity to cleanup after itself and gracefully terminate. If the process doesn't terminate after 5 wait periods (configurable, but currently 10 microseconds), it is sent a SIGKILL, gracelessly terminating it. As with traditional Linux processes, executing Tasks must have registered a cleanup method for the SIGTERM signal for the graceful shutdown to be any different from the graceless 
one.

\subsection{Example Implementation}

This section provides details on an example implementation built on top of GDMFAS. It includes derived classes to monitor resources and sample sets of Tasks that facilitate the validation of the system.

\subsubsection{CPU Resource Implementation}

The example CPU Resource implementation was created by leveraging the /proc file system to gather CPU information about both the system and the currently running process. Unlike memory usage, which can be retrieved instantaneously, CPU usage needs to be sampled to get the CPU usage of a process or the system. The pseudo-file/proc/stat/ provides the number of jiffies the system has spent in kernel mode, user mode, and the number of jiffies it has spent idling. Sampling these three values over a known period of time gives the percentages that the CPU has spent in each mode during the sample period. Similarly, reading the /proc/[PID]/stat pseudo-file gives the number of jiffies since the process started that the system has spent in each of the three modes on behalf of the tracked process optionally including the time spent on behalf of the tracked processes children. The provided CPU Resource does include time spent on behalf

of children processes. In order to get the most accurate readings of the current CPU usage, the CPU class utilizes a decaying average [3]. 


\subsubsection{Memory Resource Implementation}

Like the CPU Resource, the Memory Resource also leverages the /proc file system to gather memory information about both the system and the currently running process. For resource scaling purposes, the Memory resource is only interested in obtaining the total amount of memory available to the system and the amount of memory the tracked process is using. To gather memory information on the tracked process with process ID PID, the Memory Resource reads the /proc/[PID]/smaps pseudo-file. Unlike /proc/[PID]/stat, /proc/[PID]/smaps provides the actual memory usage of a process, instead of the maximum size it could consume.

\subsubsection{Current and Battery Resource Implementation}

The provided Current and Battery Resources are canned Resources and are not actually connected to any tracking mechanisms. Rather, both resources return example values for the purposes of GDMFAS validation. The Current Resource randomly spikes high (with a known seed) when sampled, and the Battery Resource slowly drains over time.

\subsubsection{Sample Task Sets}

To facilitate validation of the GDMFAS system, multiple process sets with different characteristics were developed to exercise the system. Included in these process sets are:

- processes with no errors with various modes of operation (i.e reading from a file and processing data or calculating values) 
- processes with policy errors (i.e those that didn't crash, but would exceed allotted resource values)

- processes with execution problems (i.e terminated as a result of segmentation faults or other signals) 


\section{Chapter 5}

\section{Validation}

This chapter discusses the validation goals, testing methodologies, and results of the GDMFAS validation efforts. It also provides an analysis of the the potential benefits and drawbacks of the framework design.

The two main validation goals of this work are verifying the correct operation of GDMFAS and gathering data on GDMFAS operation for metric $^{2}$ evaluation.

\section{$5.1 \quad$ Verifying Correctness}

In order to verify that the GDMFAS prototype functions as designed, unit tests were created to verify the GDMFAS and to verify the example implementation. These tests include exercises of the Scheduler and Task Manager, but since the System Monitor is the focus of this work's implementation and the core of the system, it is also the focus of the validation. Three different scenarios were validated using these unit tests.

1. Framework Implementation - API Correctness: Each method in the 
GDMFAS is unit tested to ensure that inputs produce the required outputs. This scenario is rather trivial, but is extremely important since any users of the system will utilize the API to construct a system.

2. Example Implementation - Nominal: The canned schedule contains no Tasks that consume resources in excess of their permitted minimums or maximums (i.e resource values are never less than 0 or greater than 1000 for non-static resources) and Tasks execute normally.

3. Example Implementation - Off-nominal: The canned schedule contains a Taskthat consumes resources in excess of its permitted maximum. The other test for this scenario contains a misbehaving processes that terminates unexpectedly (via a segmentation fault); in this case the system is expected to update Task parameters to reflect the error.

The results of these unit tests are shown in Table 5.1.

Table 5.1: Results for Unit Test Scenarios

\begin{tabular}{|c|c|c|}
\hline & Scenario & Pass /Fail \\
\hline 1 & Framework - API Correctness & Pass \\
\hline 2 & Example - Nominal & Pass \\
\hline 3 & Example - Off-nominal Resource Use & Pass \\
\hline 4 & Example - Off-nominal Misbehaving process & Pass \\
\hline
\end{tabular}

\subsection{Metric Evaluation}

This subsection provides a discussion of GDMFAS and the reasoning behind the scores assigned for the metrics presented in Chapter 2. 


\subsubsection{Extensibility}

Due to GDMFAS prototype implementation's inclusion of generic processes, inheritance, polymorphism, and default methods, the system is very Extensible. The framework interface allows the creation of new Resources through the extension of the Resource class. As long as the new Resources override a handful of methods and any Tasks that use the Resource are configured to use it, the new Resource will be seamlessly integrated into the system.

The creation of a new DMM is also equally easy to develop. Since the scheduling process iterates through all $\mathbf{N}$ of the DMM's and each returns an integer, which is subsequently summed to get the total "value" of a Task, infinitely many DMMs can be easily integrated provided that the sum of all $\mathbf{N}$ DMM scores do not exceed the maximum size of an unsigned, 32-bit integer ${ }^{1}$.

Because GDMFAS uses UDP/IP to communicate between modules, modules can moved off of the spacecraft (performance can take an enormous hit depending on what is moved) and new modules can be added without significantly modifying the code of existing modules; new commands to existing components only need to be mapped to component actions in the socket command code. This allows GDMFAS to be extended across multiple platforms at multiple different locations, the former of which is only available with ASPEN [39].

Since the majority of the system is designed in a similar fasion, GDMFAS receives a score of Very Good for the Extensibility metric.

\footnotetext{
${ }^{1} 4,294,967,295$
} 


\subsubsection{Domain Applicability}

Like CASPER and NEAT, GDMFAS was designed for and implemented in the target domain, yielding a score of Very Good for this metric.

\subsubsection{System Overhead}

During the preliminary testing of the prototype implementation, the System Monitor component used an average of $10 \%$ CPU during the execution of the tests. This value was measured using the Linux top utility during the execution of the unit tests. At this time, the GDMFAS system doesn't include the machine learning component, which will undoubtedly increase the CPU use during resource modelling. Based on prototype System Monitor measurements, GDMFAS receives a score of Very Good for the System Overhead metric. Since the Scheduler and Task Manager were not the focus of this work and were not fully implemented, a discussion on potential performance issues with those modules is provided below for the reader.

\section{Task Manager}

The largest performance concern with the Task Manager is the traversal of the dependency tree. Depending on the algorithm actually used, the overhead of doing the dependency checks can high. The use of the longestChain instance variable in the Task class is an attempt to limit the traversal overhead by having an 0 (1) check when comparing dependency tree depths of Tasks, but this instance variable will need to be set at some point. The ideal situation would be to have the system developer load in a value for the longestChain at system creation time (since the developer is perfectly aware of what needs to be accomplished and what 
the prerequisites are). Having the developer supply the maximum depth of the dependency tree may not be practical, but is not unreasonable. For the purposes of GDMFAS system, a system which is rooted in user input and customization at system creation time, the assumption that the developer will analyze the mission of the system and provide all necessary information is fundamental to the system. If the developer does not specify the longestChain at system creation time, the depth can still be calculated, but the system may incur a significant cost.

Another concern with the Task Manager is the communication format. Although the format discussed in this thesis is useful to a ground operator, it is impractical to send relatively large data over a bandwidth as low as 1200bps [20]. To remedy this, the implementation of functionality could compress the report before sending it to the ground. Since it is extremely likely that the developers will customize the mission report and communications system to suit their needs, other issues arising from a specific implementation are not considered here.

The final performance concern with the Task Manager discussed in this paper is access and storage of Tasks in memory. For the purposes of achieving O(1) access time, there exists a function in the system that maps the unique ID (id) of a Task to an index in the Task Repository. Furthermore, since the Ready Pool merely contains pointers to the Tasks in the Task Manager, there is no duplication or modification of Task data, allowing the same access properties of Tasks in the Task Repository.

\section{Scheduler}

Because the Risk Manager evaluates tasks using binary checks (is the probability of success greater than some value or not?) there aren't any real perfor- 
mance concerns with this module's default configuration. Unless the user overrides them, the default configuration only checks Task fields to make its decisions. There are very few computations executed, and even these are trivial (see Equation 3.1). As a result, the complexity of the Risk Manager (when implemented) should be 0(1).

The Prioritizer should not be a source of performance issues when it is implemented because, like the Risk Manager, it does not contain a complicated algorithm. The calculations being done in this module (in the default configuration) are trivial and merely consist of getting a Task value (such as user priority) and multiplying it by an assigned weight.

The scheduling algorithm, as outlined in this thesis, is a performance bottleneck to the same degree that sorting algorithms are bottlenecks. Given n Tasks, the Risk Manager will filter out the $\mathrm{k}$ Tasks which are too dangerous to run. Once each of the $n-k$ non-dangerous Tasks passes through the Prioritizer, it is placed on the schedule. As more Tasks are added to the schedule, they are sorted in descending order of priority. Assuming there are no Tasks with absolute deadlines, the scheduling algorithm will perform slightly better than sorting algorithms (since the algorithm terminates when the schedules length is long enough). If absolute deadlines do exist, additional checks and manipulations will need to be performed to make sure any modifications to the schedule still allow Tasks with absolute deadlines to meet those deadlines. Unlike when absolute deadlines are absent, the scheduling algorithm will need to search for the highest priority Task which will fit into the blank space on the schedule resulting from a Task ending before a Task with an absolute deadline is scheduled. For example, if a Task ends at time 1568 and a Task with an absolute deadline is scheduled to begin at 1754, the algorithm will need to find the highest priority Task that occupies less than 
or equal to 186 units of time, and thus will fit in the void. At this time, this part of the algorithm has not been fully analyzed and the exact method of making this determination has not been identified because the purpose of this work is to define a design for a framework and not an implementation for every item in the framework.

\subsubsection{Mission Accuracy}

Based on the executed unit tests, GDMFAS is able to meet absolute deadlines (such as a deployment of a sail). Although it operates correctly, the prototype scheduling algorithm is simplistic when compared against something like the NEAT scheduling algorithm [8]. As a result of its simplistic nature, it is not optimal nor real-time. In the average case, GDMFAS will not miss absolute deadlines (since it places them on the schedule first), but there may be small gaps where no Tasks can fit based on their estimated completion time. These small gaps waste time that could be spent doing useful work. Based on the properties above, the current prototype of GDMFAS receives a score of Moderate for the Mission Accuracy metric.

\subsubsection{Error Recovery}

Unlike NEAT and CASPER, GDMFAS 's error recovery system is not scheduler based. This means that GDMFAS is better suited to pre-empt misbehaving tasks before they have a chance to fail, instead of waiting until the faulty task completes

on the schedule before beginning any corrective actions like CASPER does [11]. While the selection of the replacement task in GDMFAS will not be optimal like NEAT is, the ability to prevent errors before they occur, rather than cleaning 
up the damage they cause earns GDMFAS a score of Good for the Error Recovery metric; large errors with cascading effects can be prevented and errors that do occur will leave the system in a safe state.

\subsubsection{Usability}

\section{Analysis Overview}

After the design phase of the GDMFAS was completed, a usability survey [4] was conducted to determine the complexity of the system from the viewpoint of the potential users. Additionally, the survey was used to get data on what format developers would choose for time and resource levels. In order to ensure accurate results, people with experience with the targeted systems (mobile autonomous robotic systems and satellites) were chosen as survey participants.

\section{Resource Priorities}

In order to measure the various criteria mentioned in the overview, a survey was constructed and distributed to participants via Google Docs. The survey was composed of three different parts. The first part asked the participants to rank the importance of different resources needed (power, camera, disk, memory) to take a photo of the earth from an orbiting satellite. This results from this section of the survey had several different rankings of the different resources needed for the mission. However, these rankings fell into two different categories. The first rankings scheme placed the highest priorities on the resources most related to the completion of the task. For example, when attempting to complete the task of taking a photo of the Earth, participants using the first category of rankings placed the highest priority on the camera needed to take the actual photo and the 
disk needed to store the image. Memory and power were merely an afterthought for participants falling into this category. The second ranking scheme focused more on system safety than on the completion of the task. For example, given the same task of taking a photo of the Earth, participants in making decisions according to the second ranking scheme placed the highest priorities on the power needed for the system to function and take the photo, and the disk space needed to store the photo. Although virtually all responses could be placed into these two categories, some responses were different.

\section{Data Item Clarity}

The second part of the survey asked the participants to specify field values for a given Task. The Task in the survey was to travel to some location A and take a photo of that location. This part of the survey was used to determine if the GDMFAS data items were general enough to accommodate different ideas about how to quantify various data items. Participants were required to fill in all fields of a Task, including any prerequisite chains and times. Since the design of the GDMFAS accommodates the use of different measurement units (all the framework cares about is the value, not the units) no required units are specified in this paper. As a result, there were a number of different units used on the survey. Units of time ranged from as small as milliseconds to as large as seconds. Regarding usability concerns, there was only one question in this part of the survey that was a source of potential issues. This issue was that some participants were unaware that TaskResources are intended to map 1:1 with the Resources used by the system. Some participants created new types of resources (such as the power needed for the camera) instead of taking the combined power needs for each aspect of the Task and consolidating it into a TaskResource named "Power" (assuming there 
is a Resource by this name).

With the exception of this question, every question in this part of the survey received answers on par with the expected inputs for a Task in the system. Based on this section of the survey, it is apparent that creating a Task in the GDMFAS system is fairly intuitive for the target audience. Since a Task also required TaskResources and prerequisites to be defined, it follows that if defining a Task is intuitive, then these data items are intuitive enough as well.

\section{General Usability Concerns:}

The third and final part of the survey asked the participants to specify any concerns that they had with the design of the system. Because this question was answerable via an open-ended paragraph, the responses covered a variety of issues within the system. The biggest problem participants had with the system was understanding specifics of the GDMFAS. For example, some participants were unsure whether or not the TaskResource usage levels included the usage levels of the Tasks prerequisites. Additionally, some participants felt that the system was too generic and were not sure how to interact with the portions of the system that were left up to the developer to complete. These issues were addressed by the addition of default implementations, which are thoroughly documented to guide the user with their own implementation. Despite these clarity issues, none of the participants indicated that vital modules were missing from the framework.

As a result of the usability survey, many of the issues users brought up were resolved. GDMFAS receives a score of Very Good for the Usability metric. 
Table 5.2: Metric Summary of GDMFAS

\begin{tabular}{|c|c|}
\hline Metric & Score \\
\hline Extensibility & Very Good \\
\hline Domain Applicability & Very Good \\
\hline System Overhead & Very Good \\
\hline Mission Accuracy & Moderate \\
\hline Error Recovery & Good \\
\hline Usability & Very Good \\
\hline
\end{tabular}




\section{Chapter 6}

\section{Conclusion}

This chapter provides closing remarks about this thesis, including the conclusion, future work, and thesis contributions.

\subsection{Conclusion}

This thesis provides the design details for three components of an executive system framework: the Scheduler, the Task Manager, and the System Monitor. The Scheduler is able to consider multiple different resources and their past and current levels. While the algorithm provided is not optimal (or near-optimal) and is not a hard real-time scheduler, it provides enough features that can operate on the millisecond granularity for it to be a useful module. The Task Manager provides the ability to keep track of tasks and the overall mission status, which is essential for people on the ground to gain an insight into system progress. The System Monitor, the focus of this work, provides the ability to execute and monitor processes; it also keeps track of the overall system status. These features allow the termination of misbehaving processes and ensure system safety. The features 
can also facilitate the gathering of process statistics and the troubleshooting of process issues during orbit.

\subsection{Future Work}

While this thesis implements some of the features in the design, much of the Scheduler and Task Manager have not been implemented due to time constraints. Therefore, the major component of the Future Work is the full implementation of these modules. Depending on the computational requirements, a much more effective scheduling algorithm can be designed and implemented. Aside from the full completion of the framework implementation, many useful modules and features can be easily built on top of the framework. Specifically, the ability to track process executions and behavior to determine software failure points would be extremely useful for the PolySat team. Another useful feature that can be developed on top of the framework is a more accurate mission status tracker; getting insight into internal system behavior would be useful on feature missions.

\subsection{Thesis Contributions}

This work contributes an accessible, compact, and easily extensive executive system framework. This framework provides a solution for the unique needs of

university CubeSats and provides the following features, which can significantly decrease development time:

- Task pre-emption 
- Resource modelling

- Task schedule filters

- Base classes for Resources and Tasks which encapsulate low-level mechanisms for resource tracking and process creation

- General system health status tracking via the System Monitor

In addition to the framework contributions, this work also provides an example implementation built on top of the GDMFAS that tracks common CubeSat resources, including memory and CPU.

This work also provides facilitates the development of important features such as Mission progress tracking via the Task Manager and analysis of process failures. 


\section{Bibliography}

[1] Atmel at697f-ek-mcga349 product ordering. http://components.arrow. com/part/link/na/en/AT697F-EK-MCGA349/Atmel?

[2] C++ list reference. http://www.cplusplus.com/reference/list/list/.

[3] Linux User's Manual - ps, Febrary 25 2010. Section 1, Notes Section.

[4] GDMFAS Usability Survey. https://spreadsheets.google.com/spreadsheet/viewform?authkey= CMqH2bIC\& formkey=dFh2QWYxdUxqMHg5YzR4UWVOZ3A0QUE6MQ\#gid=0, May 312011.

[5] Jason Anderson. Autonomous satellite operations for cubesat satellites. Master's thesis, Cal Poly State University:SLO, 2010.

[6] NASA Goddard Space Flight Center Advanced Architectures and Automation Branch. Agent technology development documents. http://web.archive.org/web/20000313004809/http: //agents.gsfc.nasa.gov/products.html, March 132000.

[7] Kim B. Bruce, Robert D. Cupper, and Robert L. Scot Drysdale. A history of the liberal arts computer science consortium and its model curricula. Trans. Comput. Educ., 10(1):3:1-3:12, March 2010. 
[8] Andrew Carrol and Phil Palmer. An evolutionary algorithm for near-optimal autonomous resource manage- ment. In 8th International Symposium on Artificial Intelligence, Robotics and Automation in Space, 2005.

[9] Andrew Carrol and Phil Palmer. Adaptive resource modelling for autonomous planning and scheduling. In IWPSS Proceedings, 2006, 2006.

[10] James Caska and Martin Schoeberl. Java dust: how small can embedded java be? In Proceedings of the 9th International Workshop on Java Technologies for Real-Time and Embedded Systems, JTRES '11, pages 125-129, New York, NY, USA, 2011. ACM.

[11] Steve Chien, Russel Knight, Andre Stechert, Rob Sherwood, and Gregg Rabideau. Using iterative repair to improve the responsiveness of planning and scheduling. In AIPS Proceedings, 2000, 2000.

[12] Steve Chien, Rob Sherwood, Daniel Tran, Benjamin Cichy, Gregg Rabideau, Rebecca Castano, Ashley Davies, Rachel Lee, Dan Mandl, Stuart Frye, Bruce Trout, Jerry Hengemihle, Jeff D’Agostino, Seth Shulman, Stephen Ungar, Thomas Brakke, Darrell Boyer, Jim Van Gaasbeck, Ronald Greeley, Thomas Doggett, Victor Baker, James Dohm, and Felipe Ip. The eo-1 autonomous science agent. In Proceedings of the Third International Joint Conference on Autonomous Agents and Multiagent Systems - Volume 1, AAMAS '04, pages 420-427, Washington, DC, USA, 2004. IEEE Computer Society.

[13] Teck H. Choo and Joseph P. Skura. Scibox: A software library for rapid development of science operation simulation, planning, and command tools. JOHNS HOPKINS APL TECHNICAL DIGEST, 25(2):154-162, 2004. 
[14] Cal Poly CubeSat. Cubesat design specification. http://www. cubesat. org/images/developers/cds_rev12.pdf, August 2009.

[15] Subrata Das, Raffi Krikorian, and Walt Truszkowski. Distributed planning and scheduling for enhancing spacecraft autonomy. In Proceedings of the third annual conference on Autonomous Agents, AGENTS '99, pages 422423, New York, NY, USA, 1999. ACM.

[16] Osvaldo Pinali Doederlein. The tale of java performance. Journal of Object Technology, 2(5), September 2003.

[17] ESA. Global educational network for satellite operations. http://genso. org/, May 2013.

[18] Delay Tolerant Networking Research Group. Ibr-dtn. http://www.dtnrg . org/wiki/Code, May 2013.

[19] Hank Heidt, Jordi Puig-Suari, Augustus S. Moore, Shinichi Nakasuka, and Robert J. Twiggs. Cubesat: A new generation of picosatellite for education and industry low-cost space experimentation. http://citeseerx.ist.psu . edu/viewdoc/download?doi=10.1.1.25.5693\&rep=rep1\&type=pdf, 2001.

[20] G. Hunyadi, D.M. Klumpar, S. Jepsen, B. Larsen, and M. Obland. A commercial off the shelf (cots) packet communications subsystem for the montana earth-orbiting pico-explorer (merope) cubesat. In Aerospace Conference Proceedings, 2002. IEEE, volume 1, pages 1-473 - 1-478 vol.1, 2002.

[21] Mark James. Jpl software download service. https://download.jpl .nasa. gov/ops/request/request_introduction.cfm, 2013.

[22] M. Tim Jones. Inside the linux 2.6 completely fair scheduler. http://public.dhe.ibm.com/software/dw/linux/ 
l-completely-fair-scheduler/l-completely-fair-scheduler-pdf . pdf, 2009.

[23] Andrew Kalman. Hardware and software design of an msp430-based satellite using an rtos. http://www.cubesatkit.com/docs/press/pumpkin_ MSP430_ATC2004.pdf, 2013.

[24] Bryan Klofas. Cubesat communication systems 2003-2013: A historical look. http://www.klofas.com/papers/GroundStation_Workshop_ Klofas_CubeSat_History.pdf, April 232013.

[25] Brian Knosp. Science and technology: Up, up, and away. http: //scienceandtechnology.jpl.nasa.gov/newsandevents/newsdetails/ ?NewsID=1910, May 62013.

[26] Milton L. Lavin and Jeanna S. Makihara. The JPL Software Development Process Description (JPL D-15378 ). JPL, revision d edition, November 15 1999.

[27] Robert Lougher. Jamvm at sourceforge. http://jamvm. sourceforge.net/, January 2010.

[28] Greg Manyak. Fault tolerant and flexible cubesat software architecture. Master's thesis, Cal Poly State University:SLO, 2011.

[29] NASA. Overview of the glory mishap investigation results for public release. http://www.nasa.govOverview/pdf/728836main_T9_MIB_Public_ Release_Summary.pdf, 2013.

[30] The Linux Kernel Organization. Linux 2.6.23 kernel source. https://www. kernel.org/pub/linux/kernel/v2.6/linux-2.6.23.tar.gz, 2007. 
[31] Chandandeep Singh Pabla. Completely fair scheduler. Linux J., 2009(184), August 2009.

[32] Barney Pell, Douglas E. Bernard, Steve A. Chien, Erann Gat, Nicola Muscettola, P. Pandurang Nayak, Michael D. Wagner, and Brian C. Williams. An autonomous spacecraft agent prototype. In Proceedings of the first international conference on Autonomous agents, AGENTS '97, pages 253-261, New York, NY, USA, 1997. ACM.

[33] PolySat. More dbs: Welcome to more dbs. http://moredbs atl.calpoly. edu/, 2009.

[34] PolySat. Polysat homepage. http://polysat.calpoly.edu/, 2013.

[35] et al. Robert L. Staehle. Interplanetary cubesats: Opening the solar system to a broad community at lower cost. http://www.nasa.gov/pdf/ 716078main_Staehle_2011_PhI_CubeSat.pdf, December 82012.

[36] Mehran Sahami, Steve Roach, Ernesto Cuadros-Vargas, and David Reed. Computer science curriculum 2013: reviewing the strawman report from the acm/ieee-cs task force. In Proceedings of the 43rd ACM technical symposium on Computer Science Education, SIGCSE '12, pages 3-4, New York, NY, USA, 2012. ACM.

[37] Gene Sally. Embedded java with gcj. http://www.linuxjournal.com/ article/8757, March 302006.

[38] Daniel Selva and David Krejci. A survey and assessment of the capabilities of cubesats for earth observation. http://systemarchitect.mit.edu/docs/ selva12b.pdf, December 2011. 
[39] R. Sherwood, A. Govindjee, D. Yan, G. Rabideau, S. Chien, and A. Fukunaga. Using aspen to automate eo-1 activity planning. In Aerospace Conference, 1998 IEEE, volume 3, pages 145 -152 vol.3, mar 1998.

[40] GCC Team. The gnu compiler for the javatm programming language. http: //gcc.gnu.org/java/, April 2013.

[41] GENIE Development Team. Genie homepage. http://aaaprod.gsfc. nasa.gov/gensaa/genie/, July 2009.

[42] Walt Truszkowski, Harold Hallock, and James Kurien Point of contact. Agent technology from a nasa perspective, 1999.

[43] Directorate of Defense Trade Controls U.S. Department of State. Us state department - policy. http://pmddtc.state.gov/regulations_laws/itar_ official.html, April 12012. 